\title{
Phase-Transfer Catalysis via a Proton Sponge: A Bifunctional Role for Bis-Cyclopropenimine
}

Lee Belding, Peter Stoyanov, Travis Dudding*

Brock University, 500 Glenridge Avenue, St Catharines, ON L2S 3A1

Table of Contents:

1. Results from the extraction-type phase transfer reactions $\quad$ S2

2. ${ }^{1} \mathrm{H},{ }^{13} \mathrm{C}$, and NMR spectra. S3

3. DFT calculated geometries and thermochemical data $\quad$ S27 


\section{Results from the extraction-type phase transfer reactions}

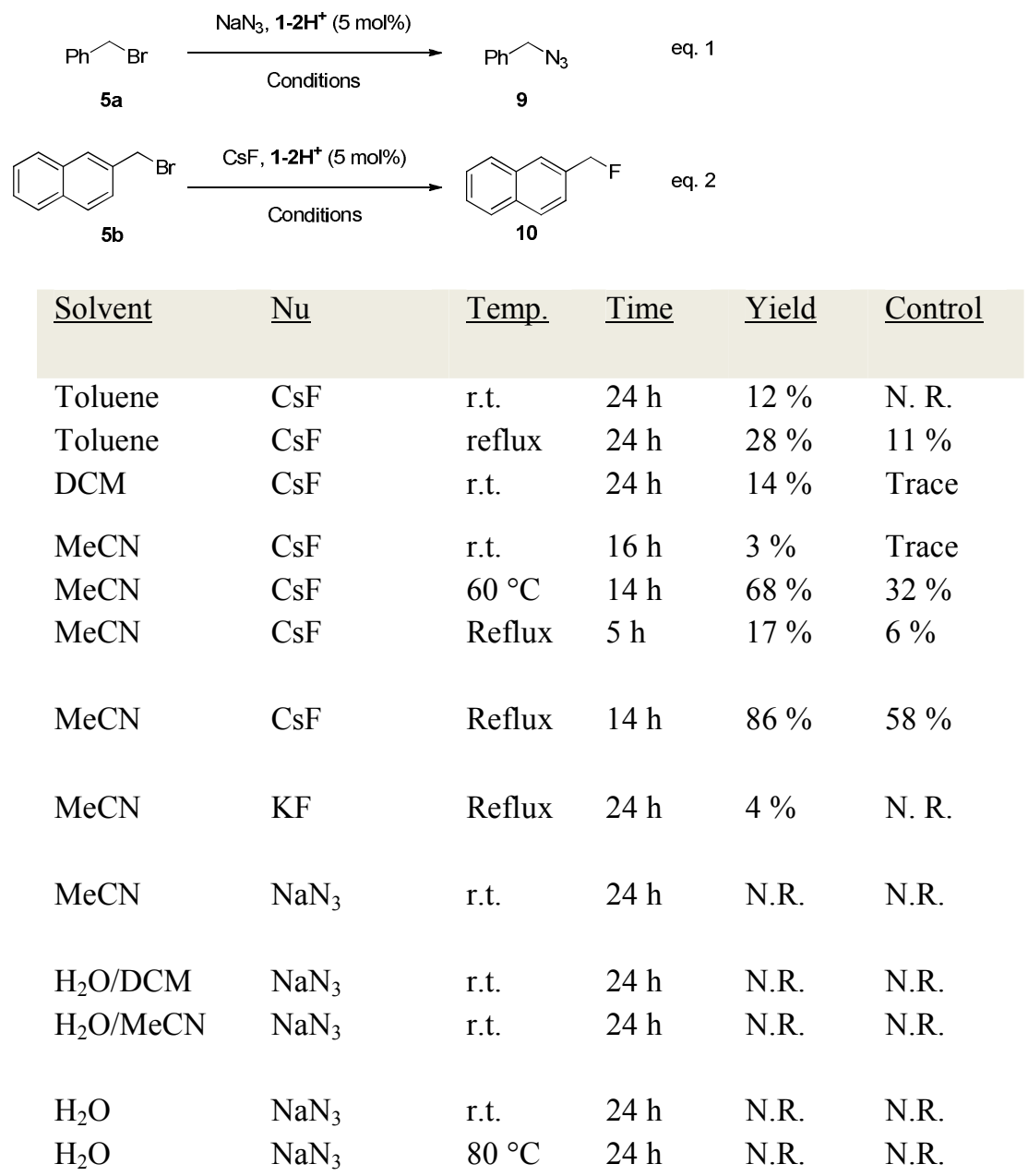


6a. ${ }^{1} \mathrm{H},{ }^{13} \mathrm{C}$, and NMR spectra.

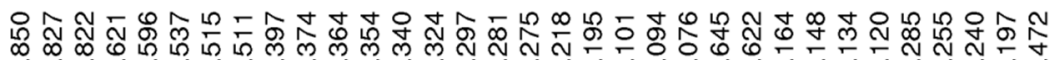

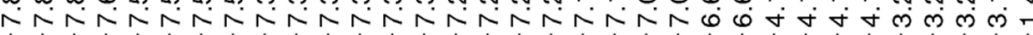

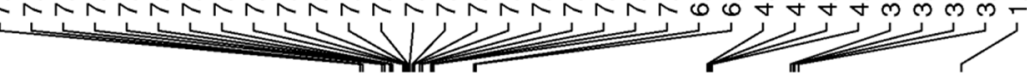<smiles>CCCOC(=O)C(Cc1ccccc1)N=C(c1ccccc1)c1ccccc1</smiles>

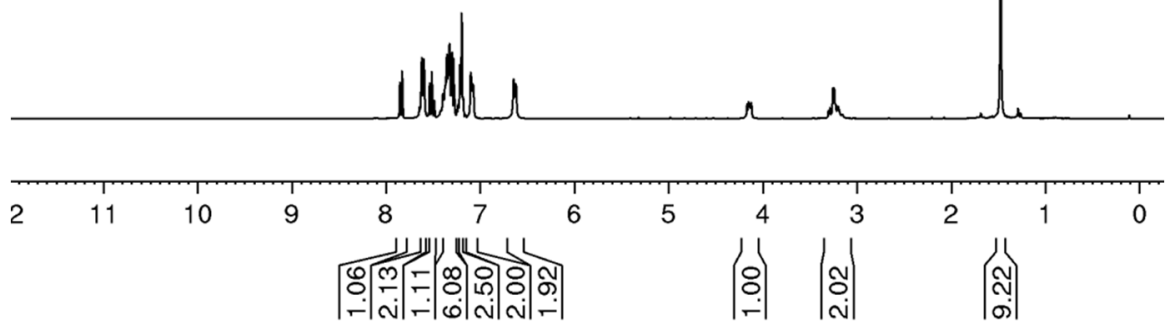




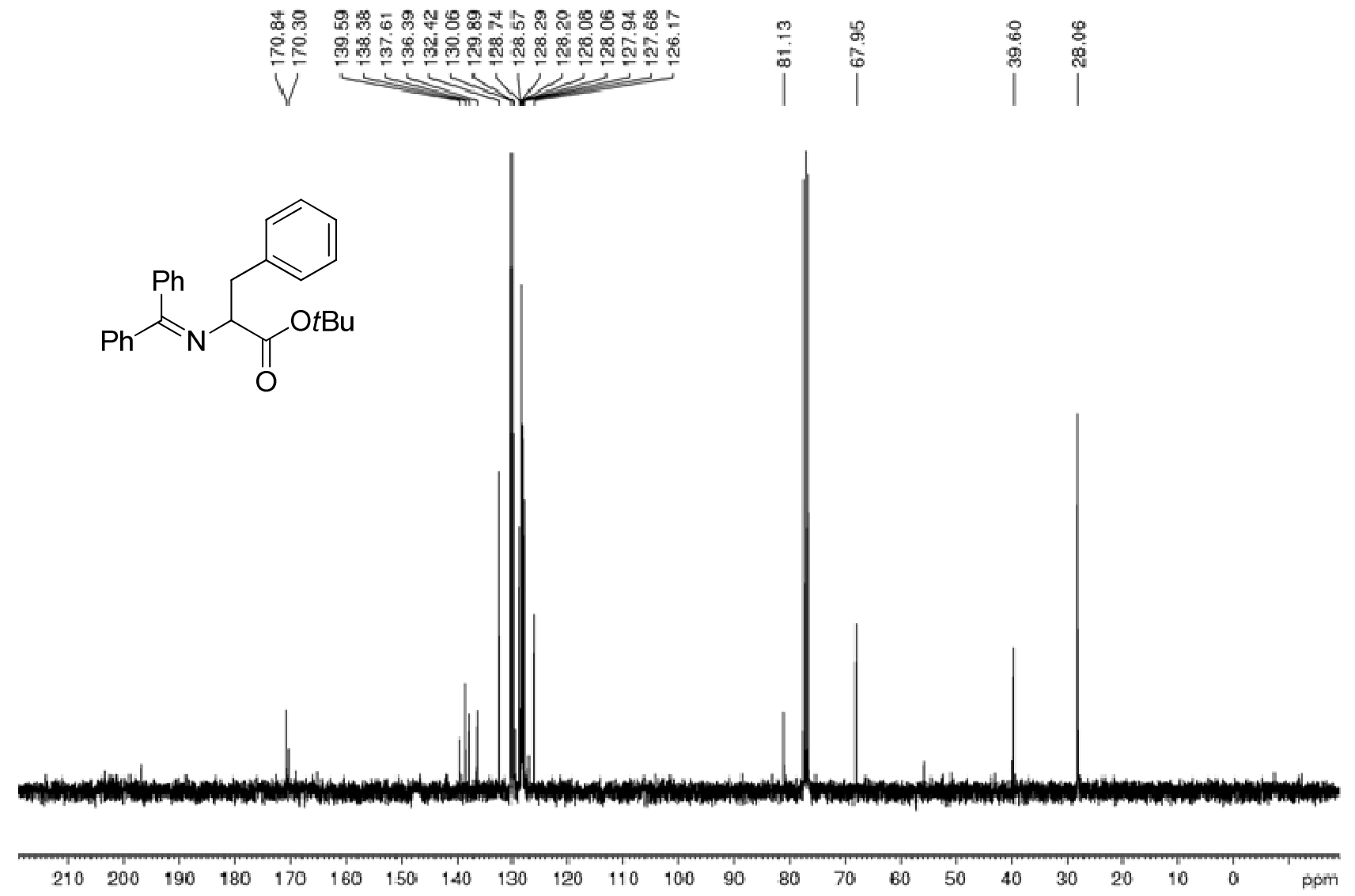


6d. ${ }^{1} \mathrm{H}$ NMR spectrum.

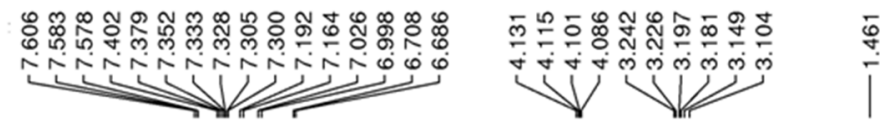<smiles>CCCOC(=O)C(Cc1ccccc1)N=C(c1ccccc1)c1ccccc1</smiles>

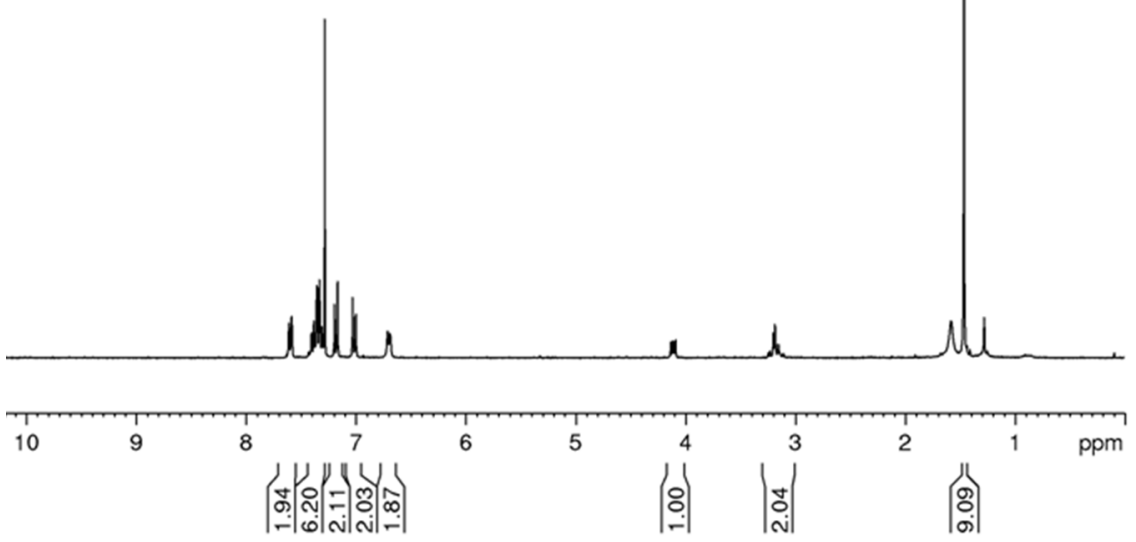


6b. ${ }^{1} \mathrm{H},{ }^{13} \mathrm{C}$, and NMR spectra.

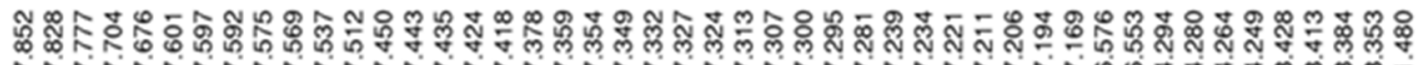

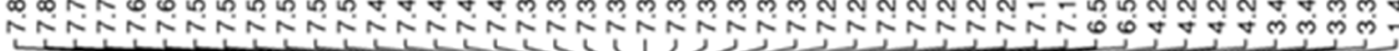<smiles>CCOC(=O)C(Cc1ccc2ccccc2c1)N=C(c1ccccc1)c1ccccc1</smiles>

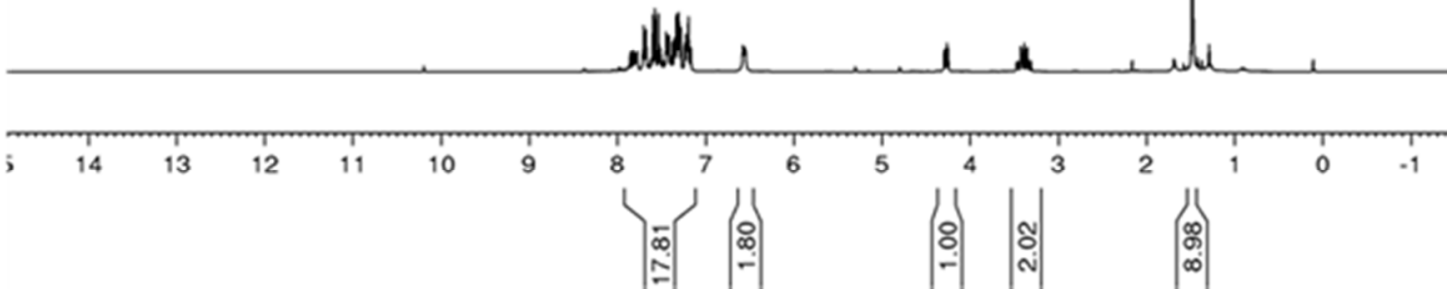



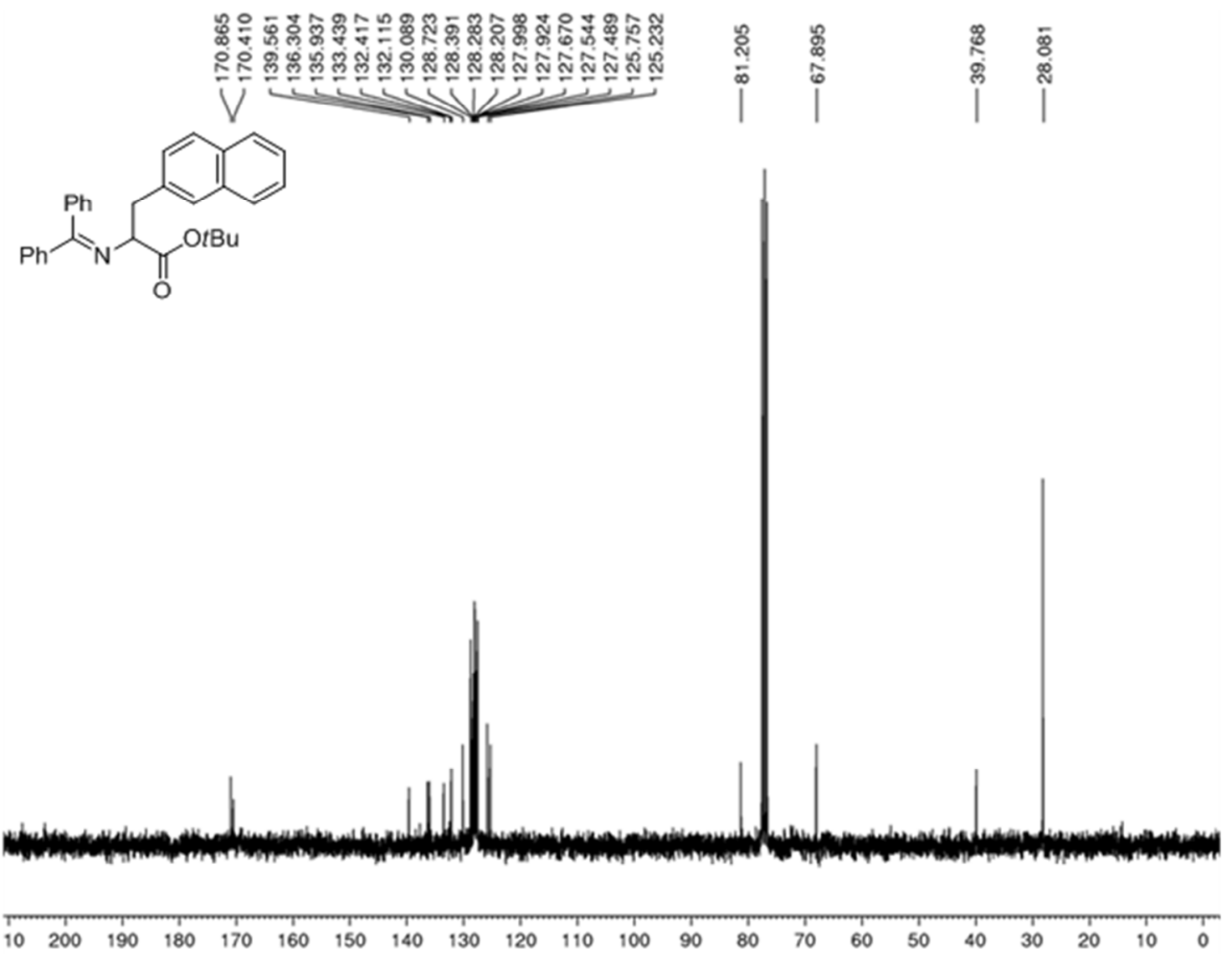
6c. ${ }^{1} \mathrm{H},{ }^{13} \mathrm{C}$, and NMR spectra.

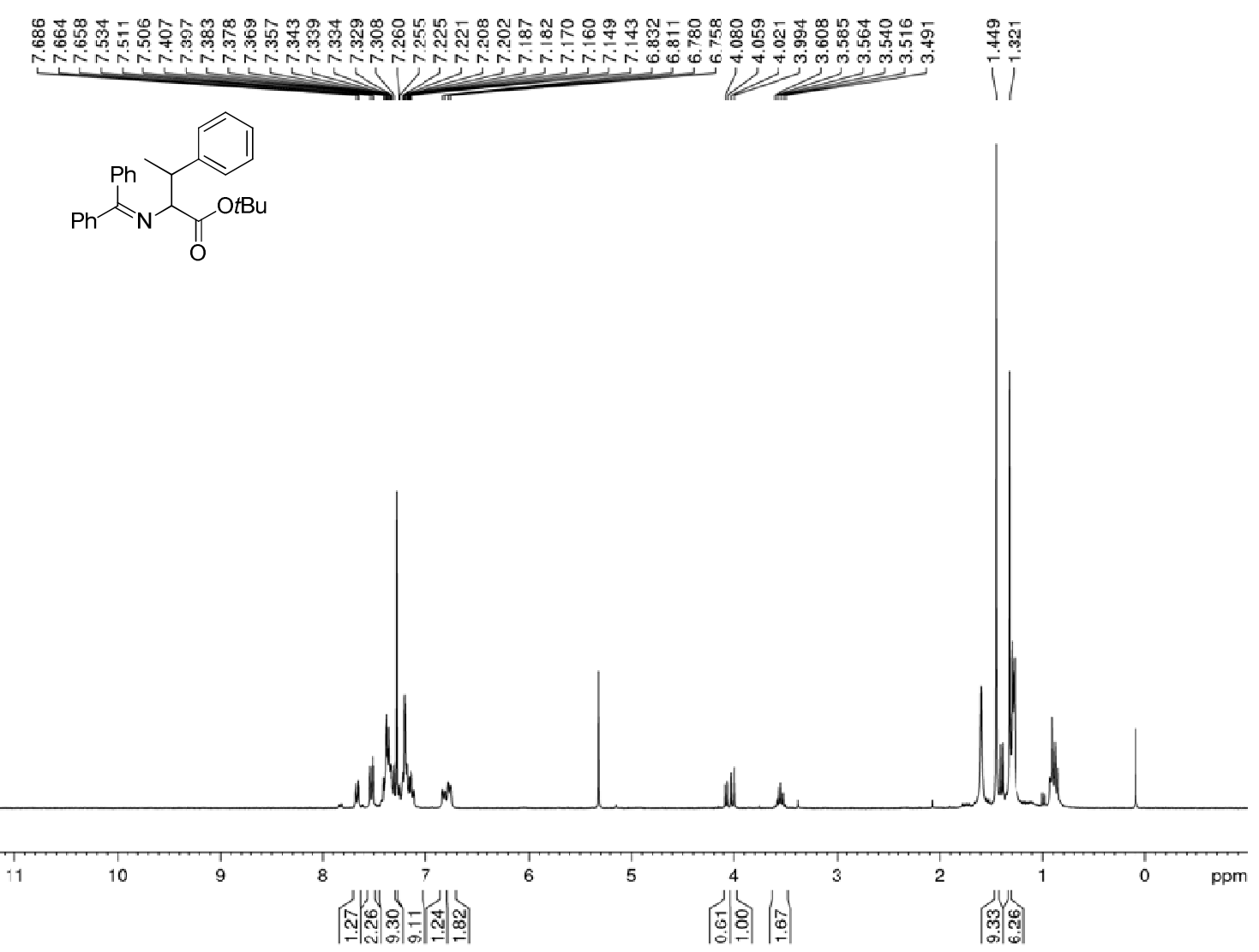




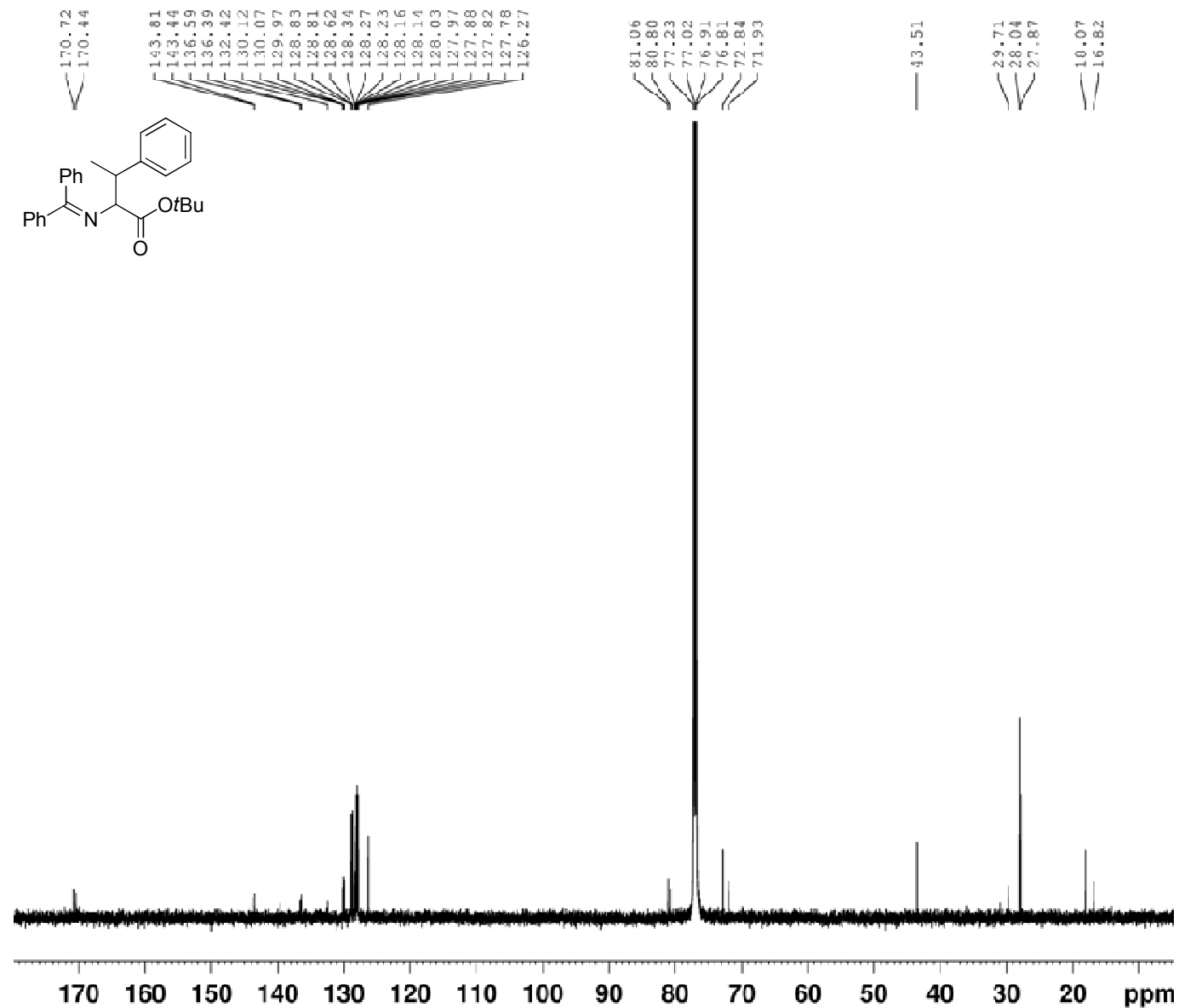


6f. ${ }^{1} \mathrm{H},{ }^{13} \mathrm{C}$, and NMR spectra.
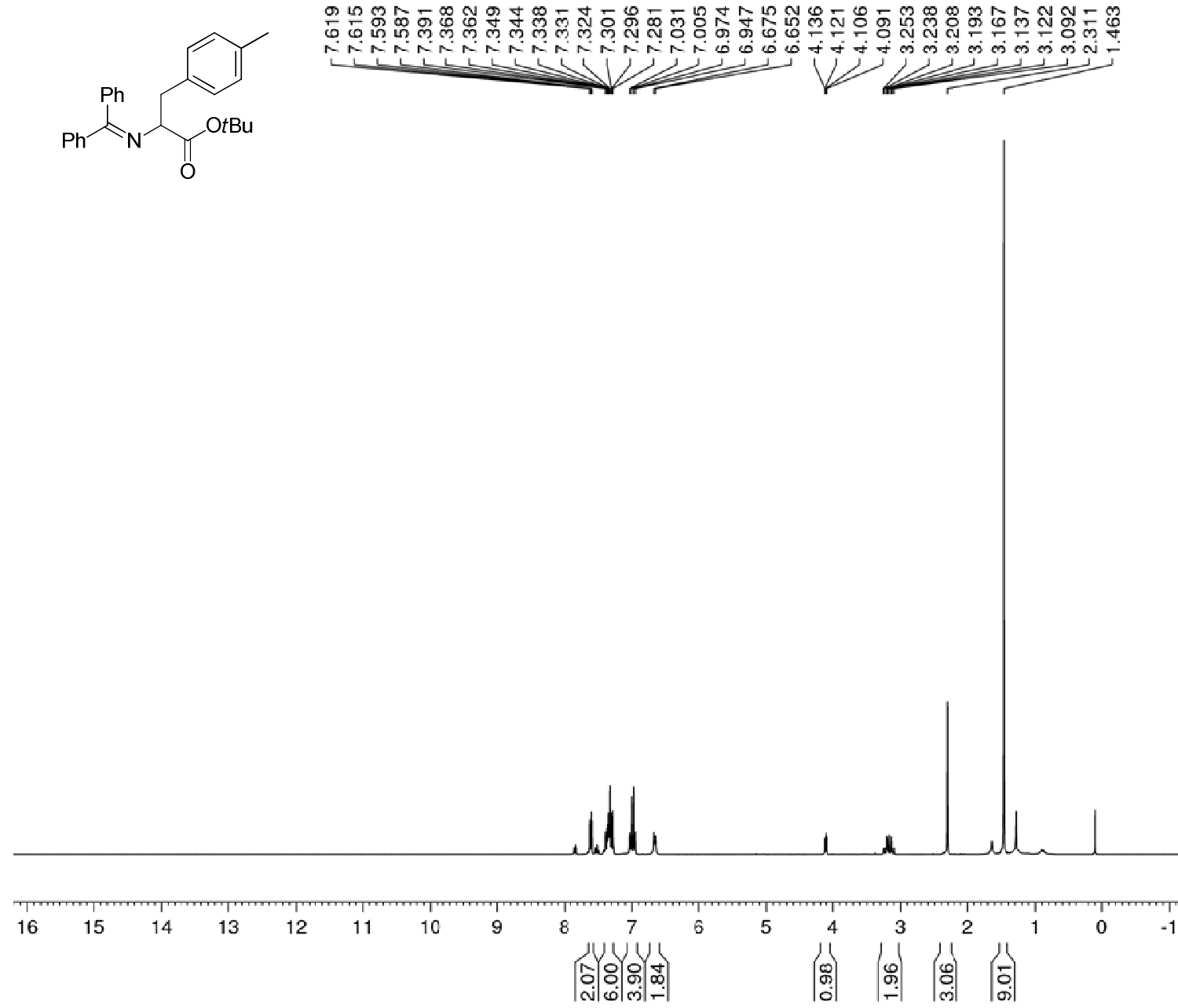

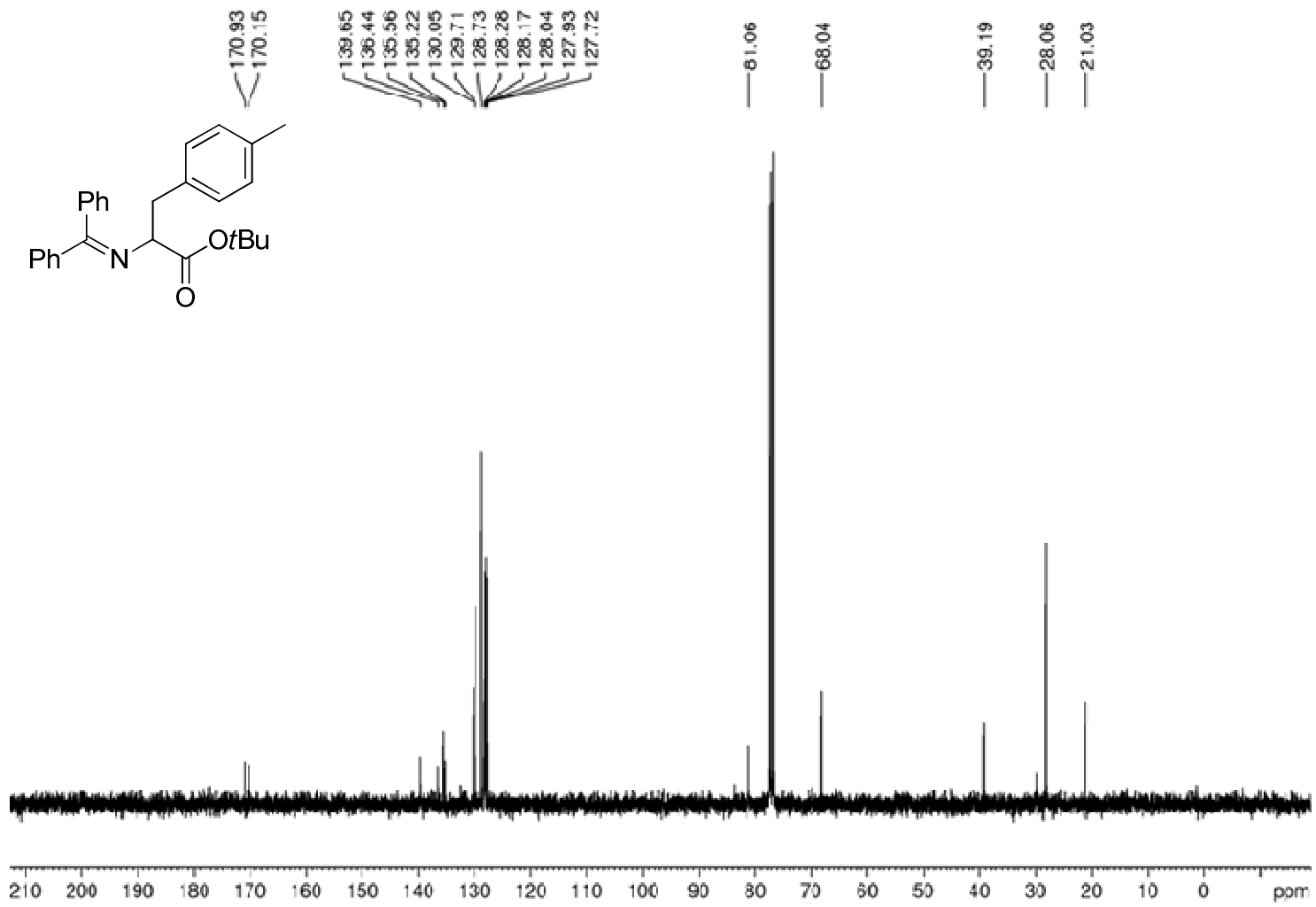
6g. ${ }^{1} \mathrm{H},{ }^{13} \mathrm{C}$, and NMR spectra.
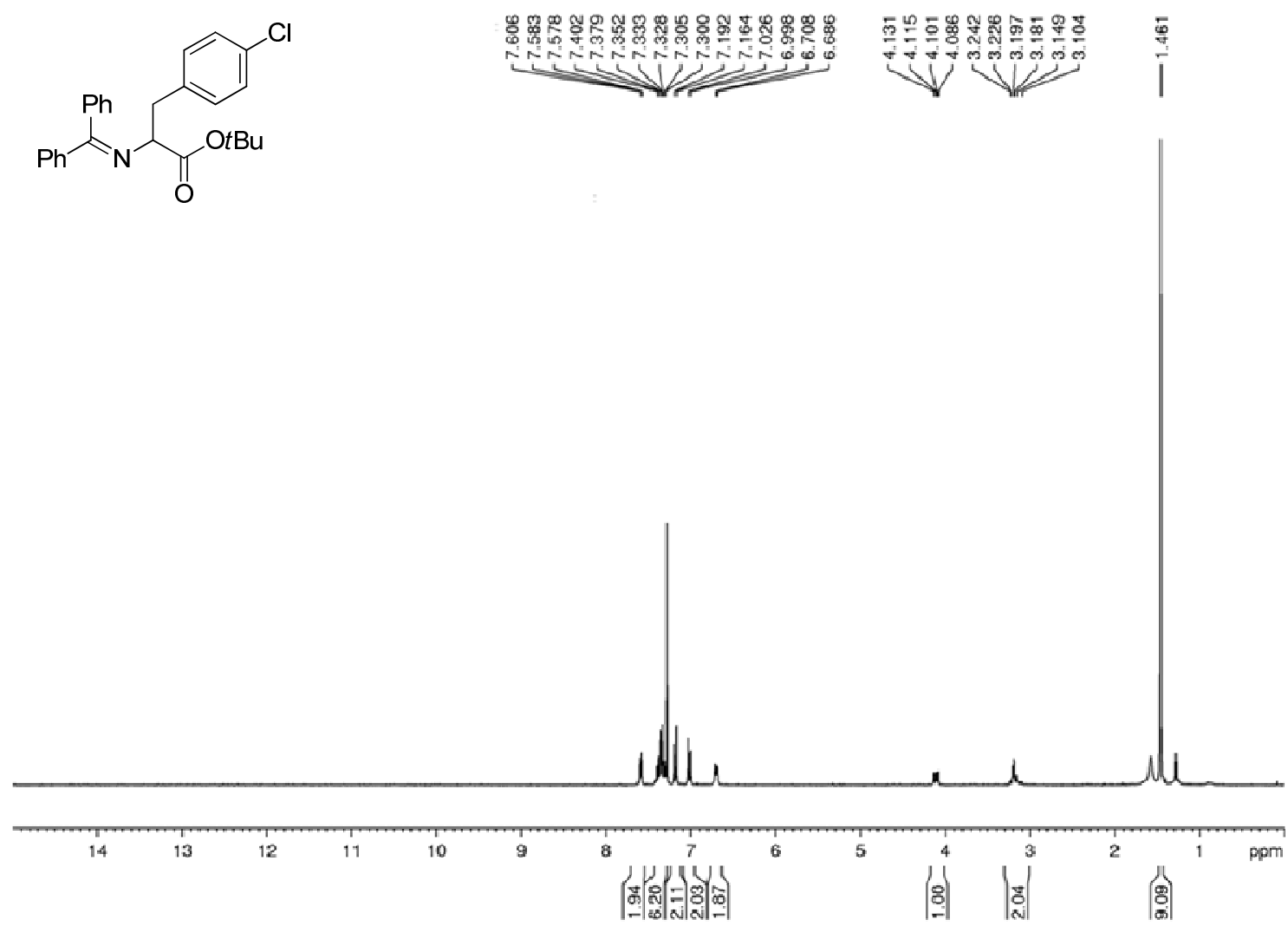

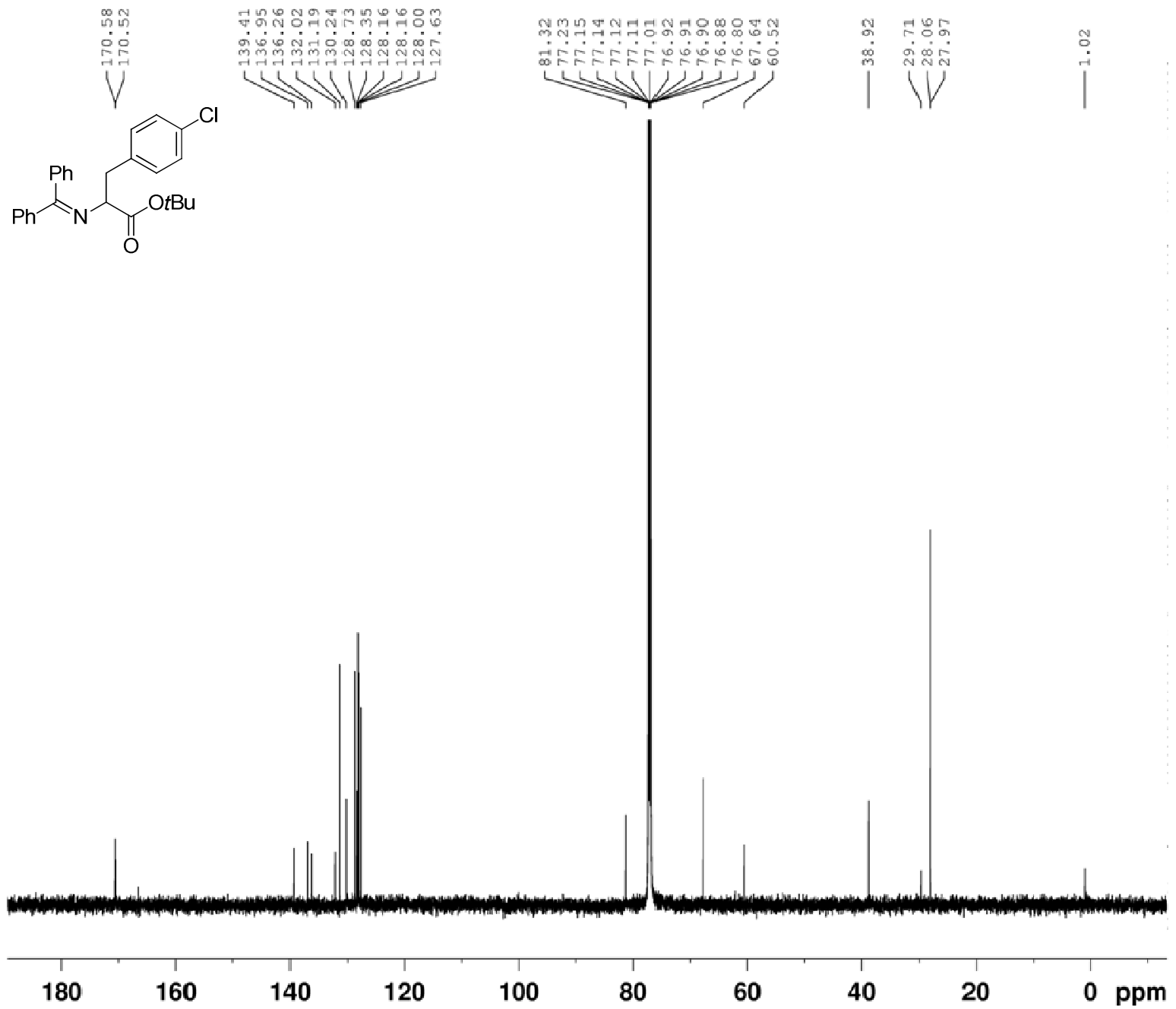
6h. ${ }^{1} \mathrm{H},{ }^{13} \mathrm{C}$, and NMR spectra.
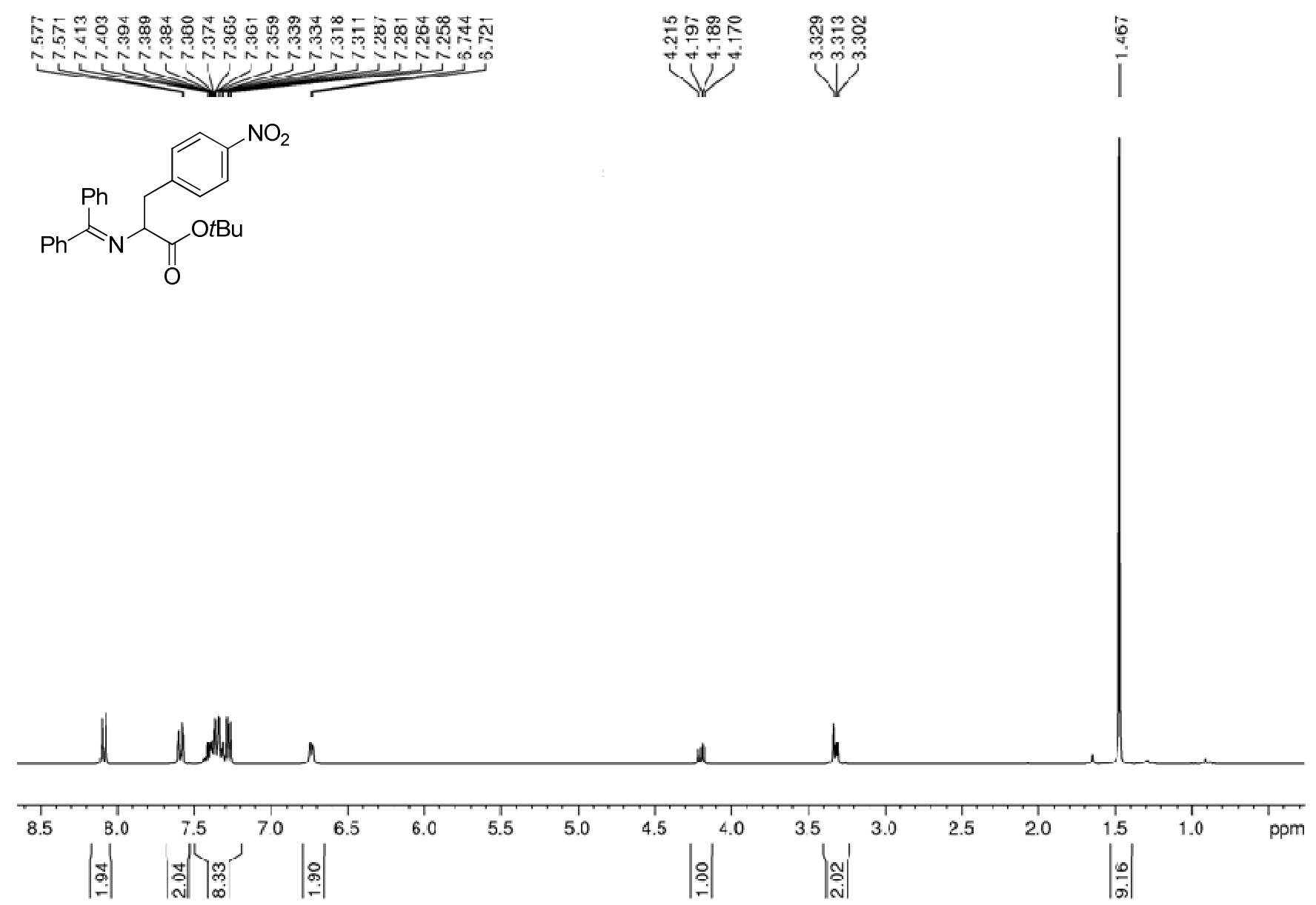


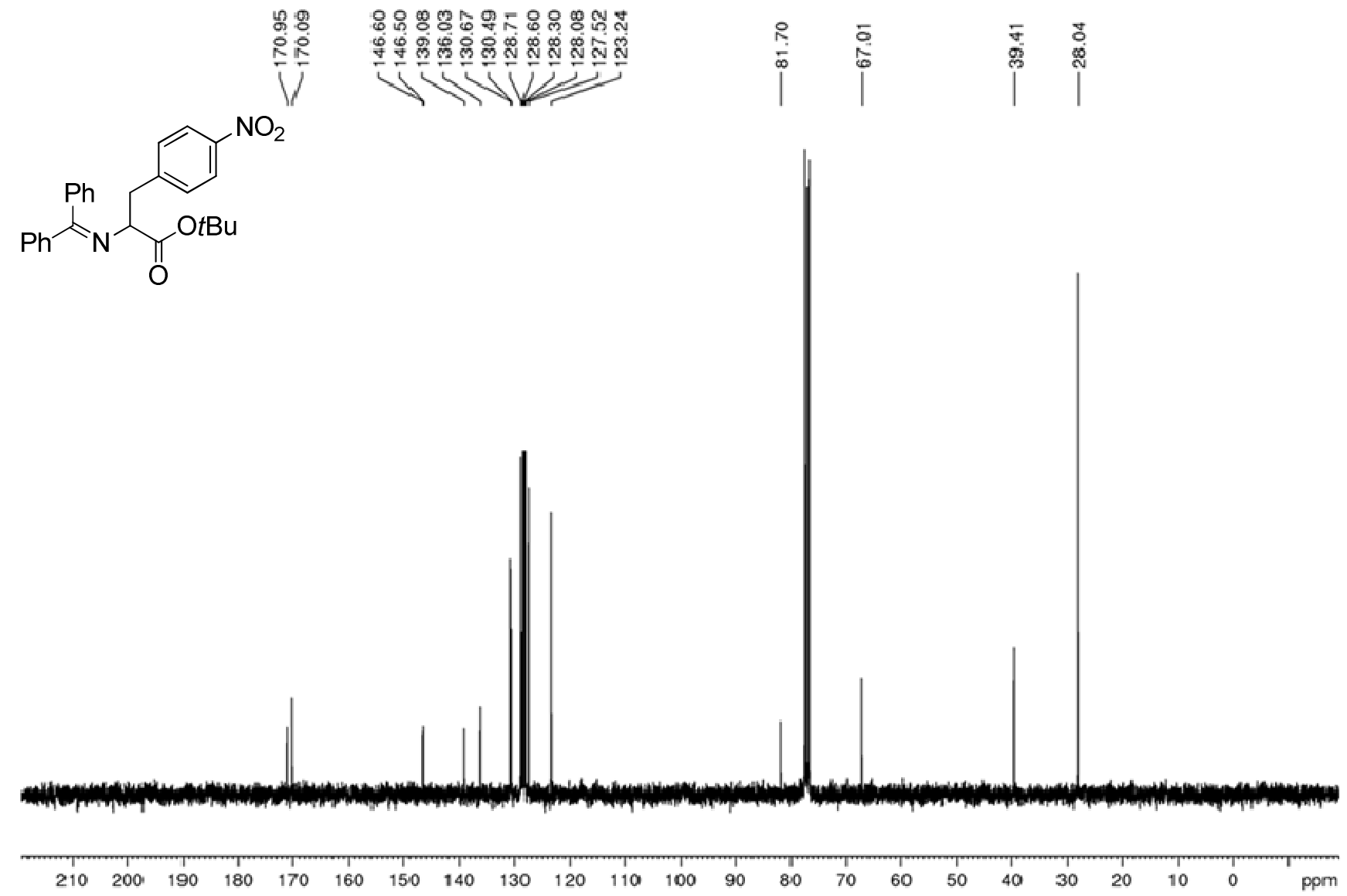


6e. ${ }^{1} \mathrm{H},{ }^{13} \mathrm{C}$, and NMR spectra.

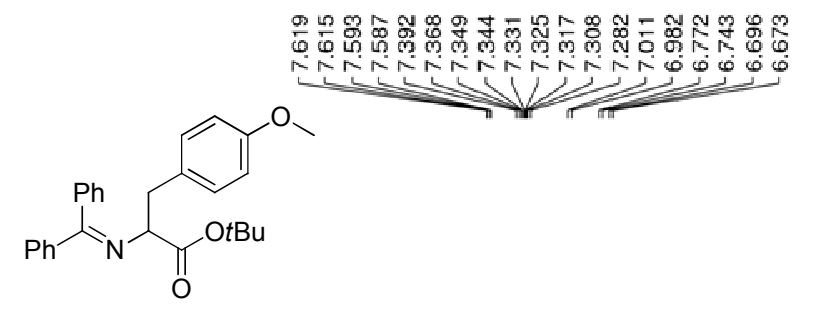

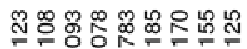

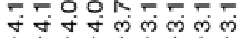

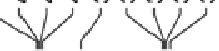

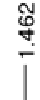

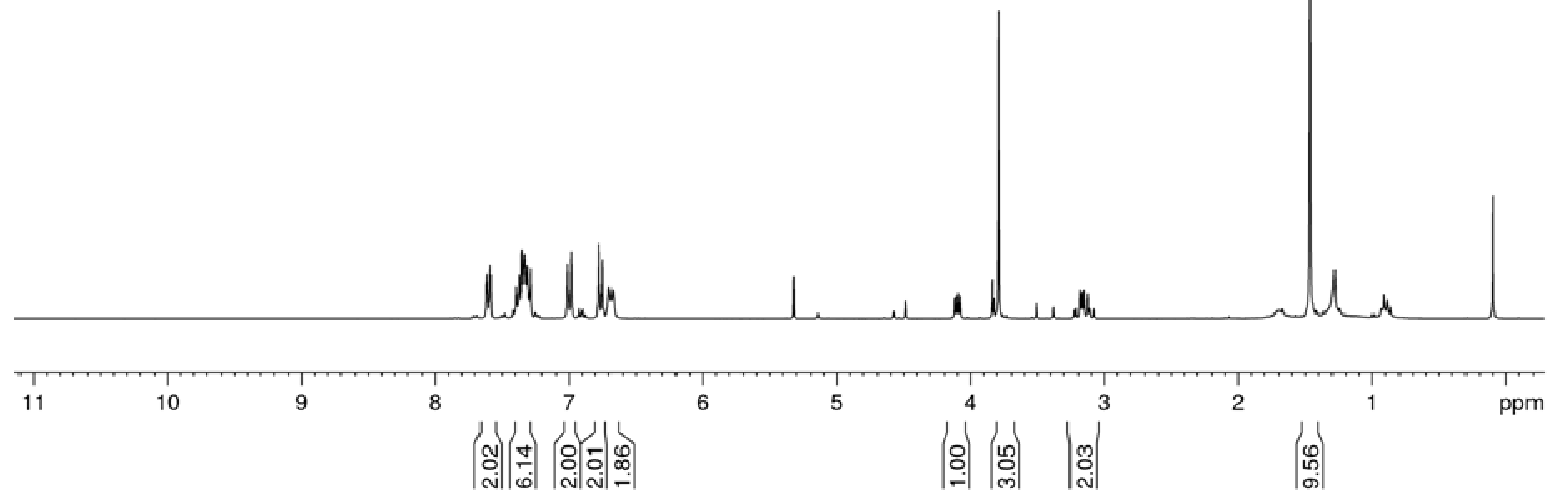



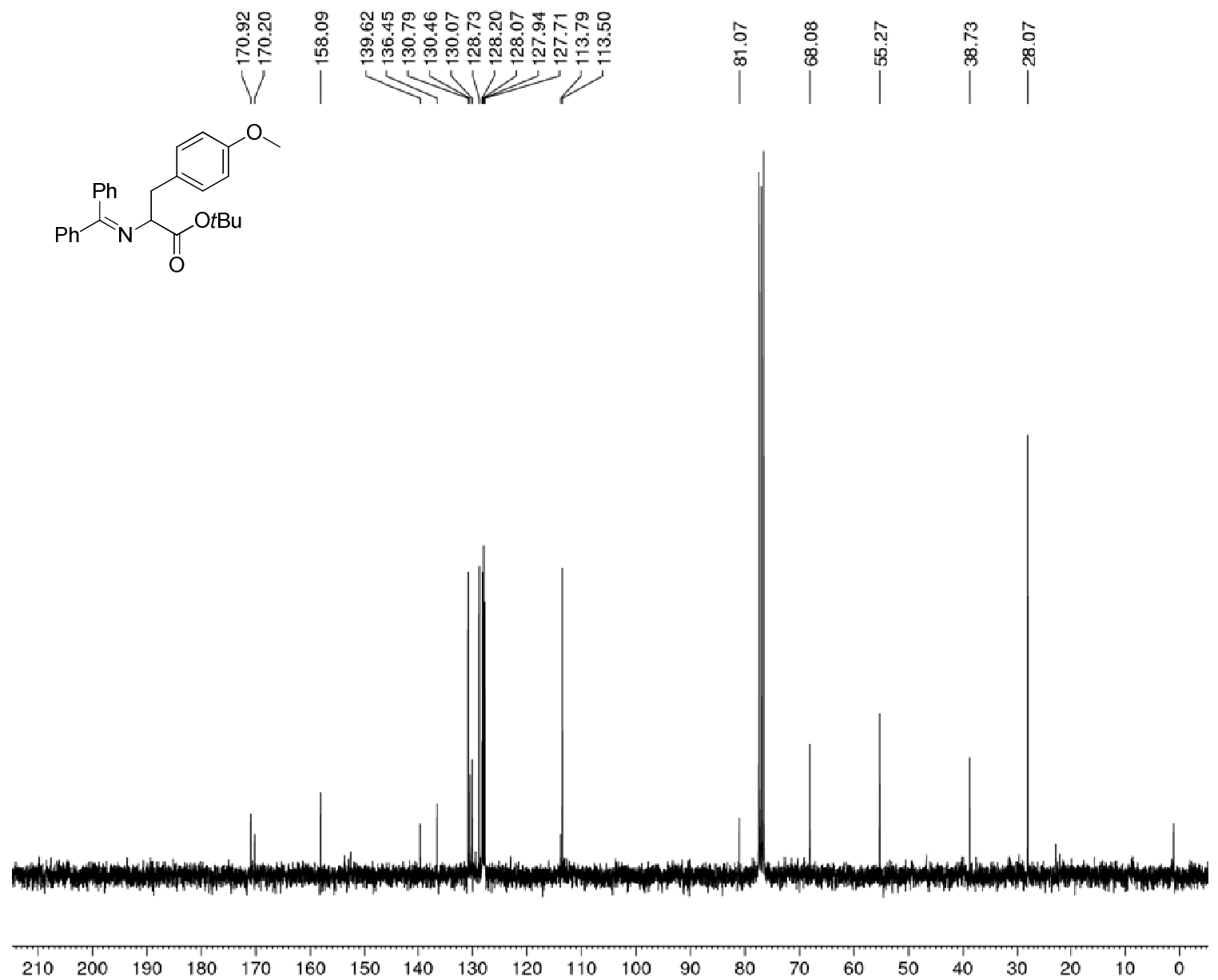
6i. ${ }^{1} \mathrm{H},{ }^{13} \mathrm{C}$, and NMR spectra.

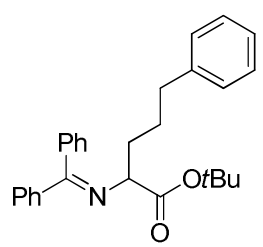

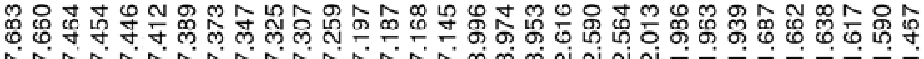
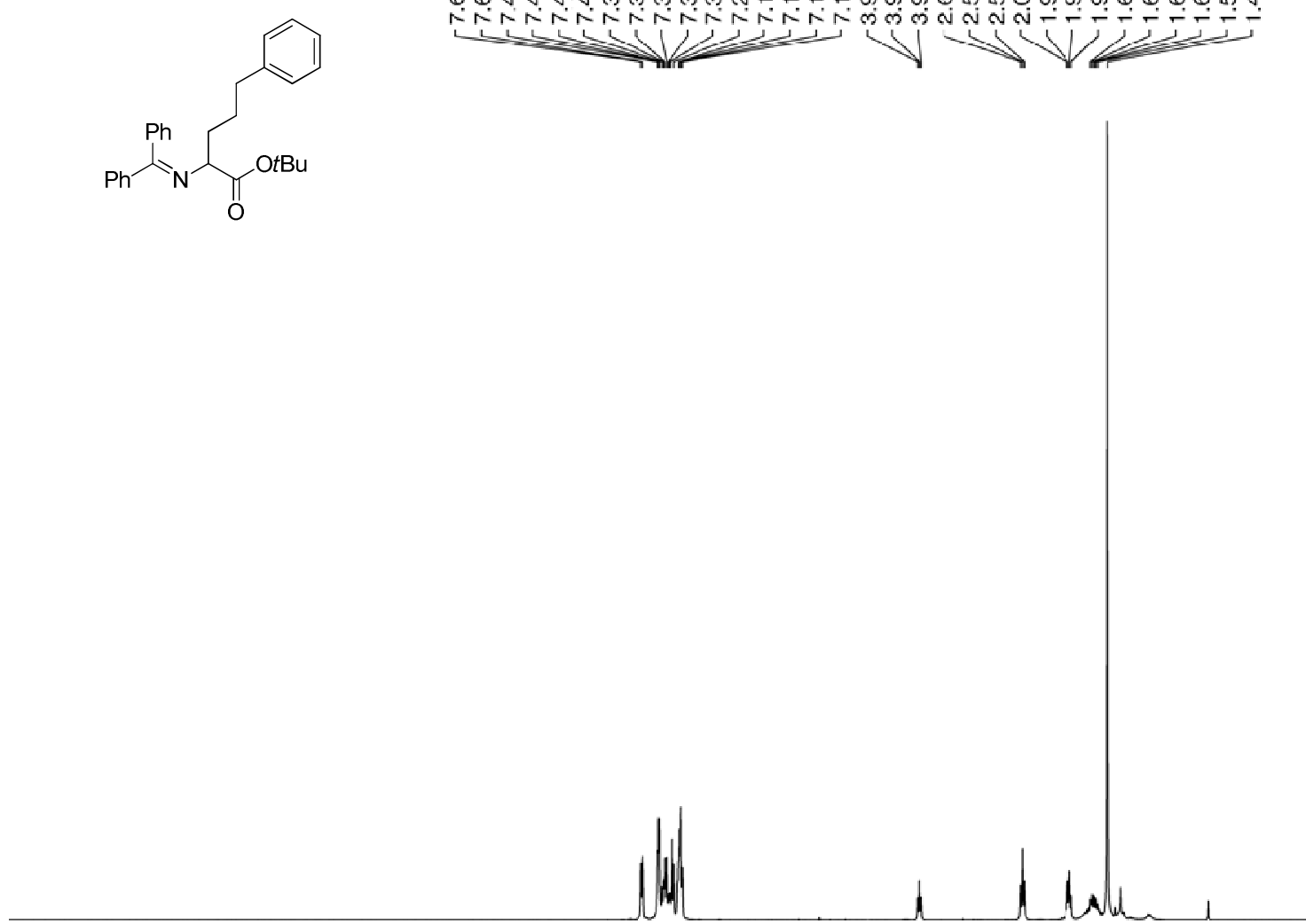

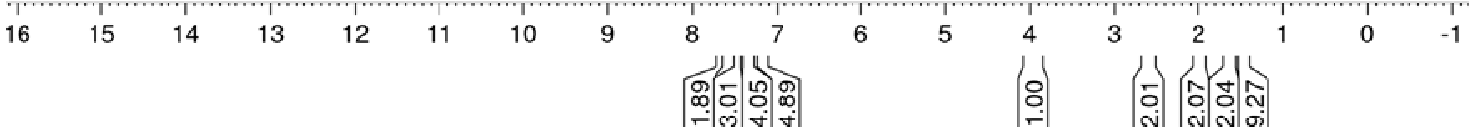




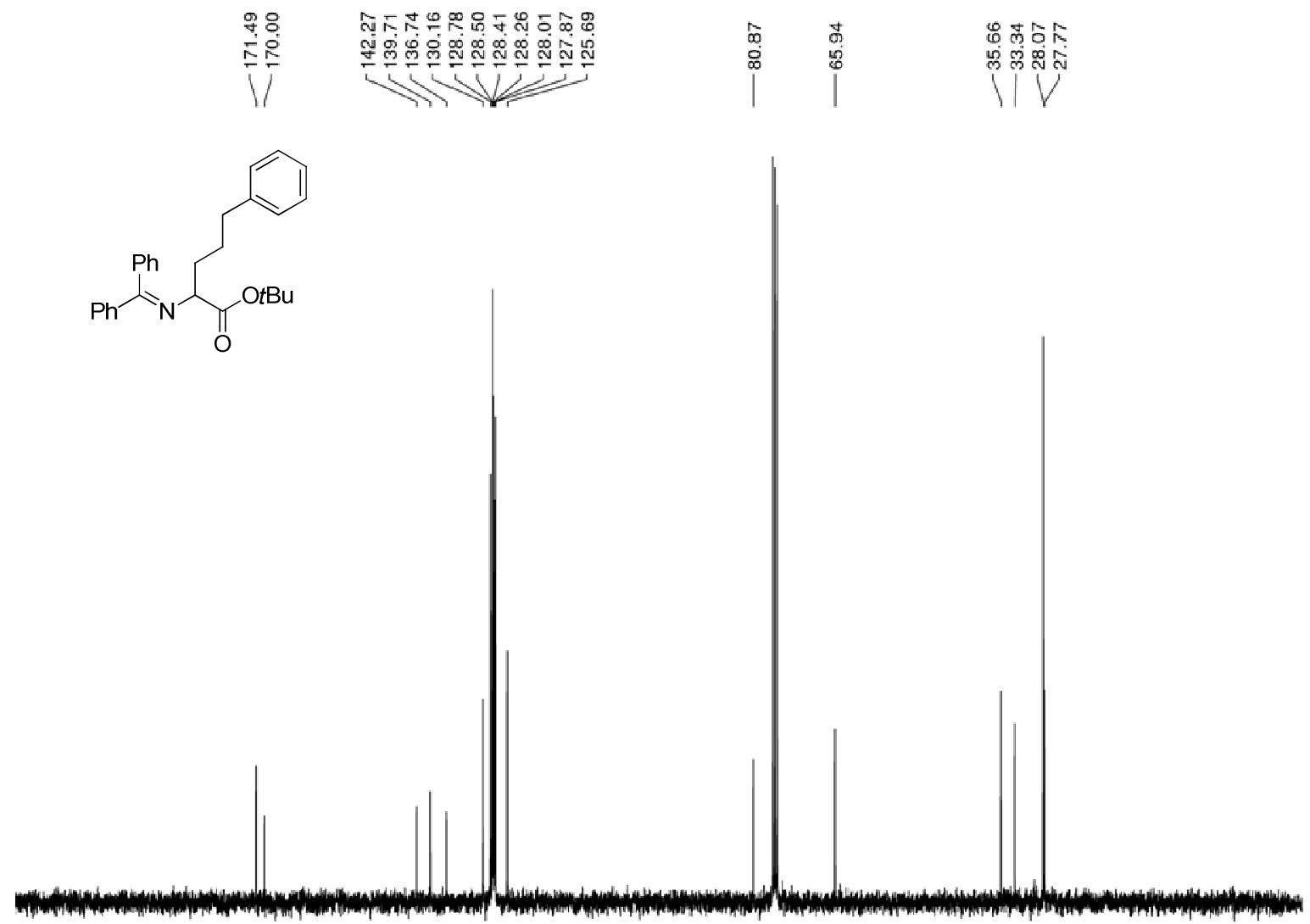

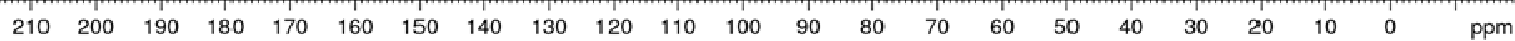


6j. ${ }^{1} \mathrm{H},{ }^{13} \mathrm{C}$, and NMR spectra.

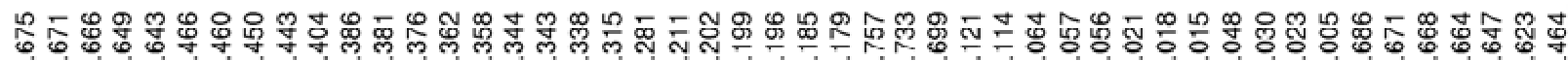

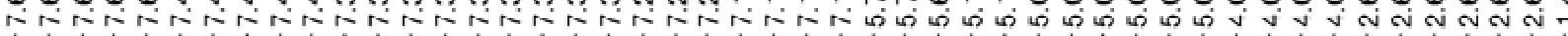<smiles>C=CCC(N=C(c1ccccc1)c1ccccc1)C(=O)OCCC</smiles>

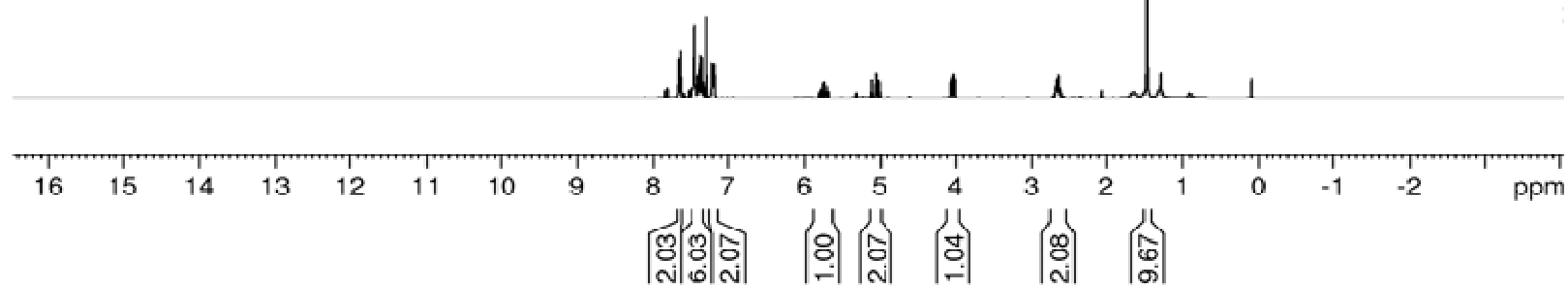



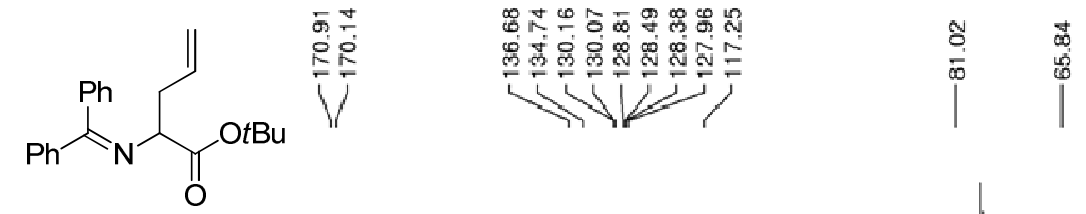

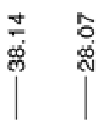

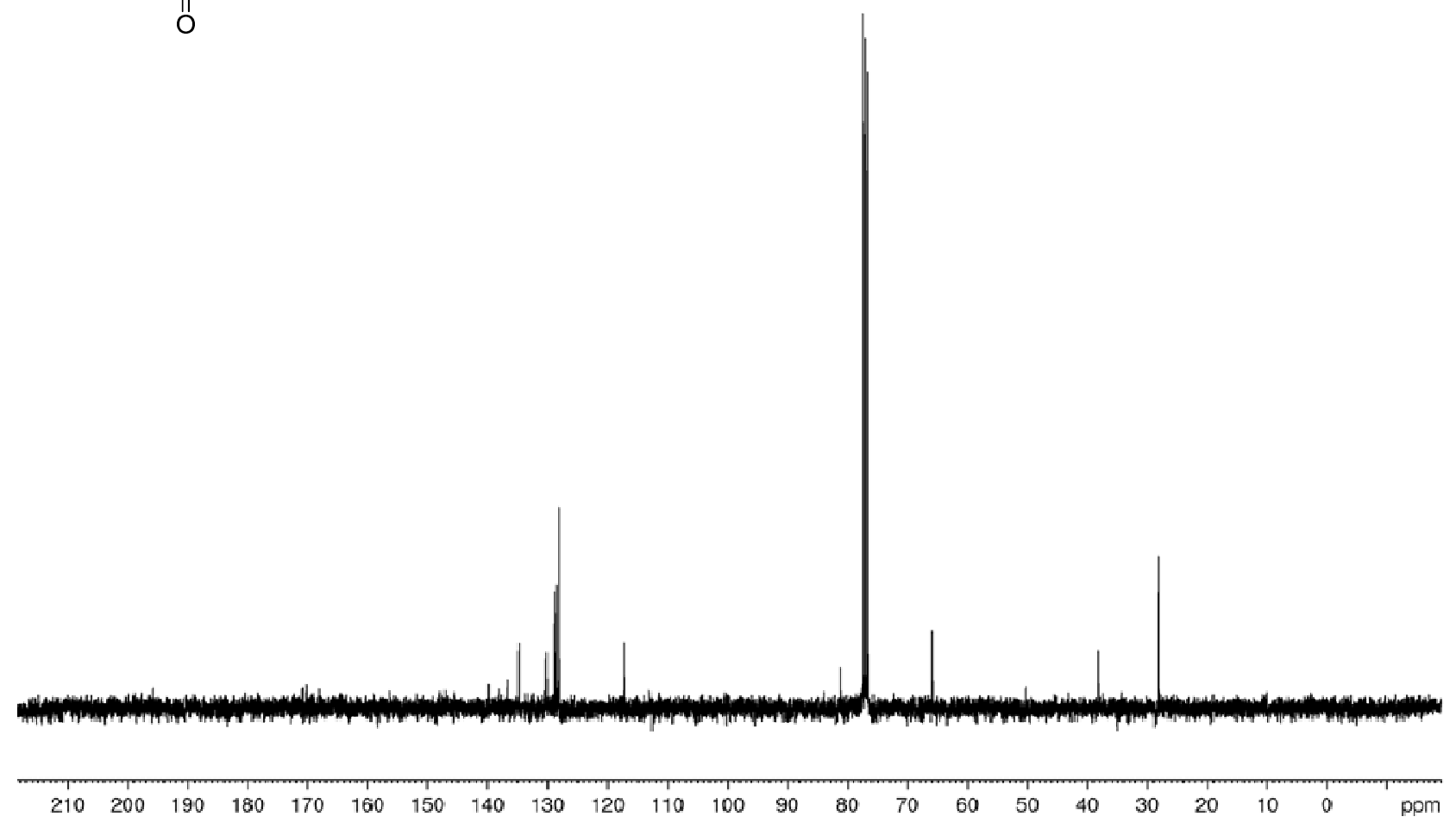


6k. ${ }^{1} \mathrm{H}$ NMR spectrum.

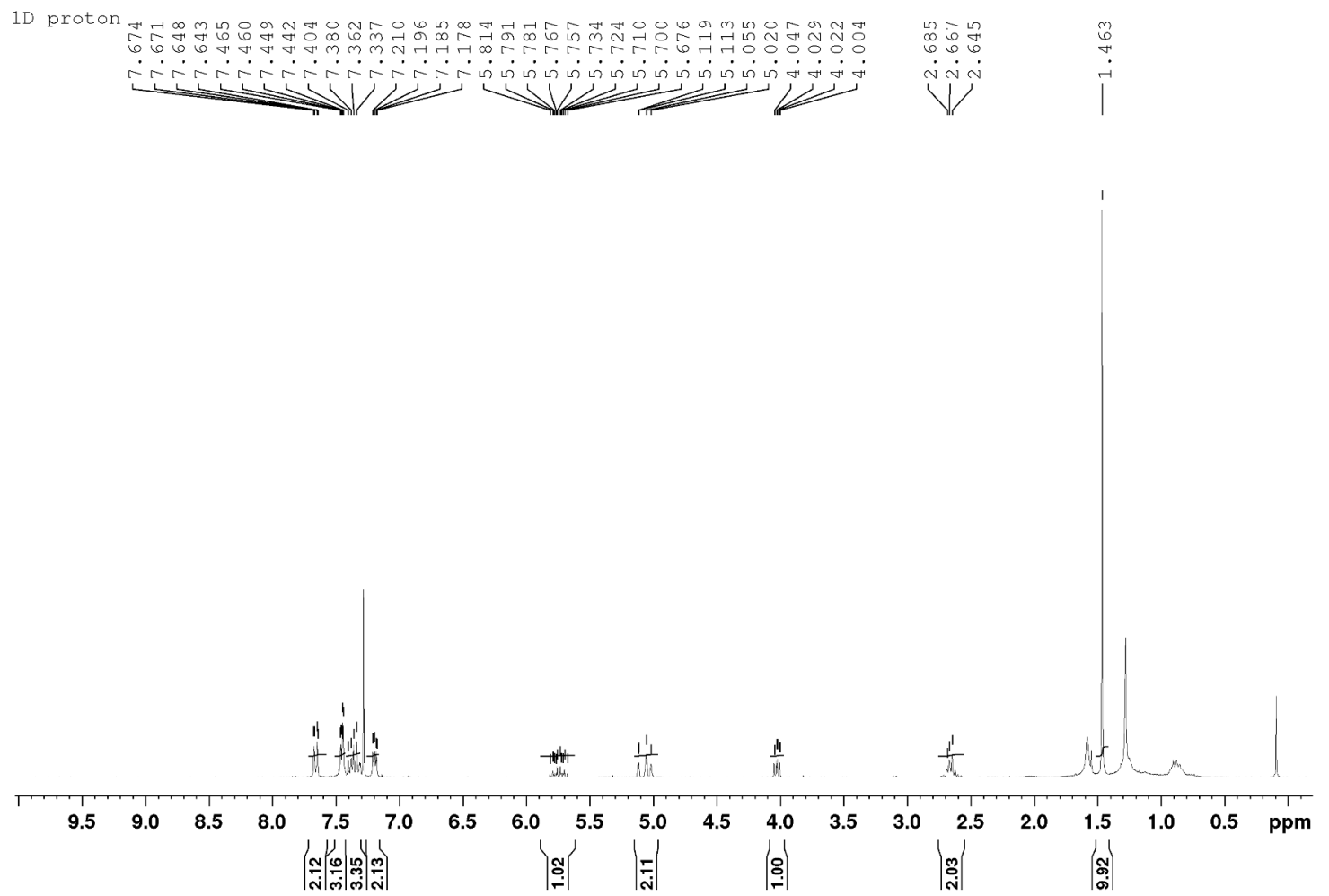


6i. ${ }^{1} \mathrm{H},{ }^{13} \mathrm{C}$, and NMR spectra.

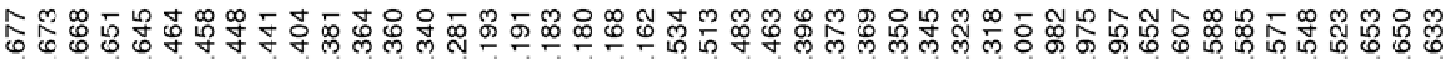

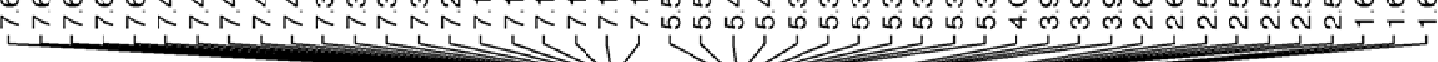<smiles>C/C=C/CC(N=C(c1ccccc1)c1ccccc1)C(=O)OCCC</smiles>
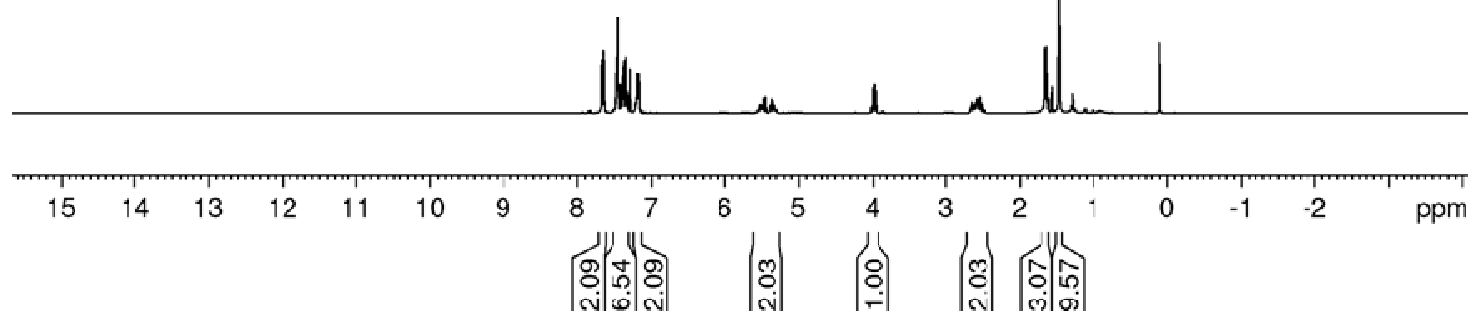


$$
\text { | }
$$<smiles>C/C=C/CC(N=C(c1ccccc1)c1ccccc1)C(=O)OCCC</smiles>

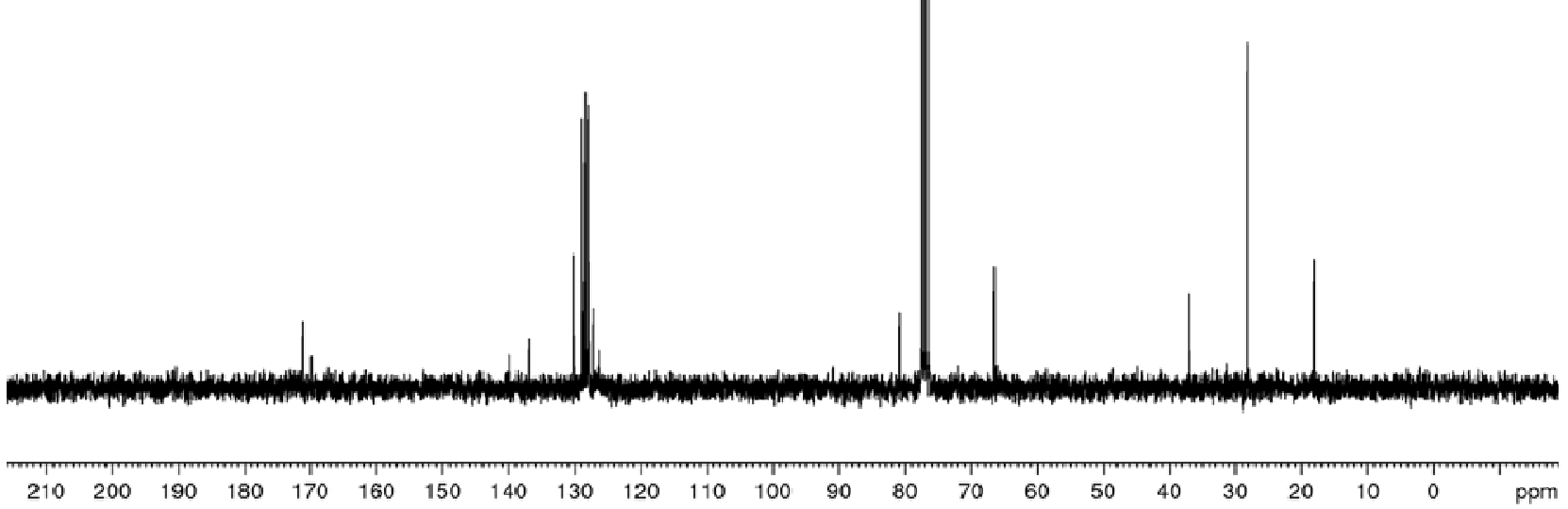


6m. ${ }^{1} \mathrm{H},{ }^{13} \mathrm{C}$, and NMR spectra.
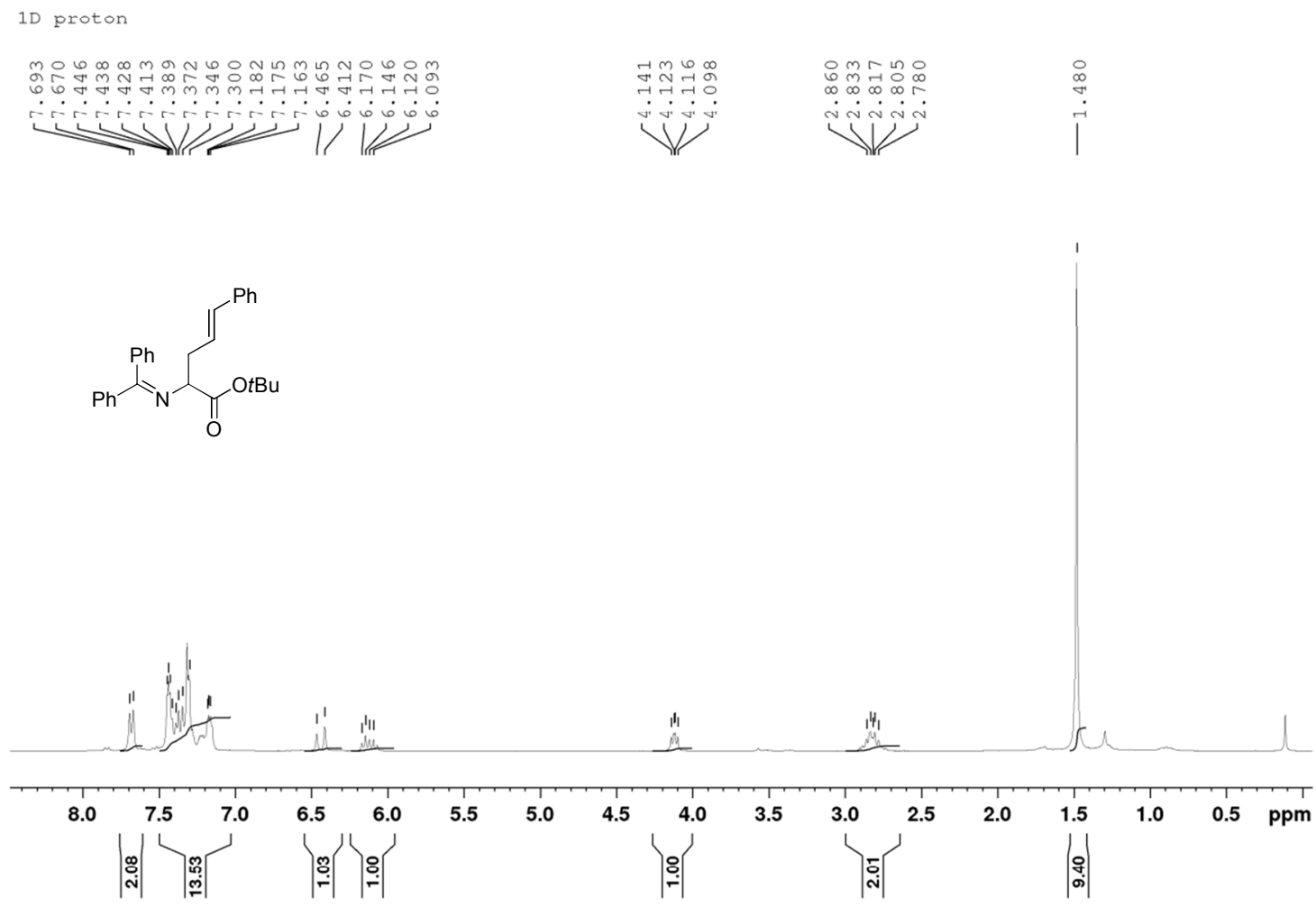

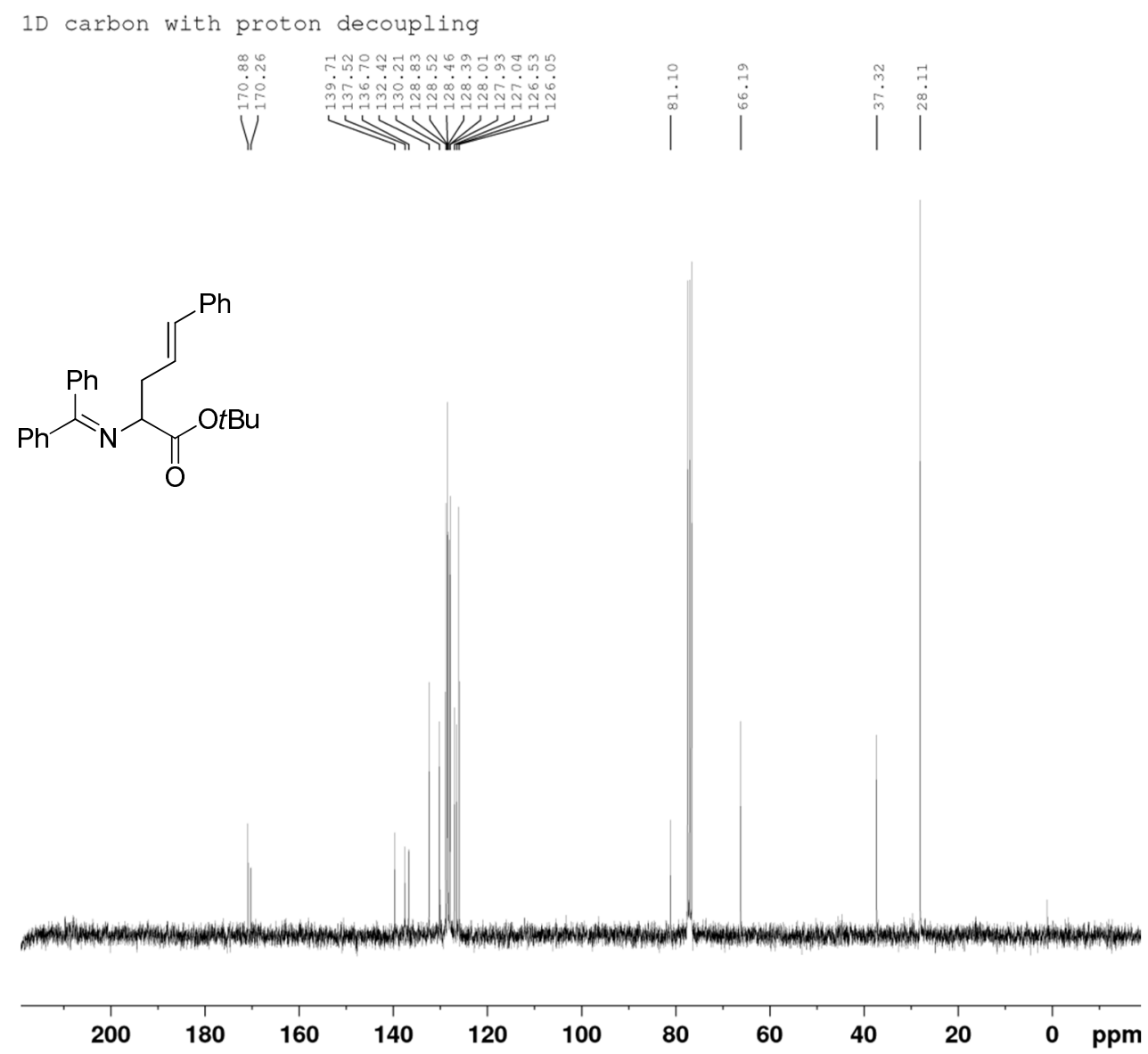


\section{Coordinate and thermochemical data for all computed structures}

Enolate, $\mathbf{4}^{-}$.

\begin{tabular}{|c|c|c|c|c|c|c|c|}
\hline \multicolumn{3}{|c|}{ Zero-point correction $=$} & \multicolumn{2}{|c|}{0.349480 (Hartree/Particle) } & & & \\
\hline \multicolumn{3}{|c|}{ Thermal correction to Energy $=$} & \multicolumn{2}{|c|}{0.369633} & & & \\
\hline \multicolumn{3}{|c|}{ Thermal correction to Enthalpy $=$} & \multicolumn{2}{|c|}{0.370577} & & & \\
\hline \multicolumn{5}{|c|}{ Thermal correction to Gibbs Free Energy $=\quad 0.299469$} & & & \\
\hline \multicolumn{5}{|c|}{ Sum of electronic and zero-point Energies $=\quad-940.720026$} & & & \\
\hline \multicolumn{5}{|c|}{ Sum of electronic and thermal Energies $=\quad-940.699873$} & & & \\
\hline \multicolumn{5}{|c|}{ Sum of electronic and thermal Enthalpies $=\quad-940.698929$} & & & \\
\hline \multicolumn{5}{|c|}{ Sum of electronic and thermal Free Energies $=$} & & & \\
\hline-11 & & & & $\mathrm{C}$ & -2.66382500 & 4.11304100 & -0.12688600 \\
\hline $\mathrm{O}$ & 2.94168700 & -2.24927000 & 0.16640100 & $\mathrm{H}$ & -4.58396800 & 3.21400700 & -0.49476000 \\
\hline $\mathrm{C}$ & 2.26387700 & -1.21556500 & 0.08701600 & $\mathrm{H}$ & -0.61673200 & 4.67777300 & 0.24411300 \\
\hline $\mathrm{N}$ & 0.14797400 & -0.05749500 & -0.00172400 & $\mathrm{H}$ & -3.03546700 & 5.13273700 & -0.15831900 \\
\hline $\mathrm{C}$ & -1.15740200 & 0.07493500 & -0.00873700 & $\mathrm{O}$ & 2.83683200 & 0.02801900 & 0.00906900 \\
\hline $\mathrm{C}$ & -2.09818300 & -1.08436800 & 0.01018000 & $\mathrm{C}$ & 4.26786000 & 0.19479200 & 0.00538800 \\
\hline $\mathrm{C}$ & -3.06904900 & -1.22525900 & 1.01197600 & $\mathrm{C}$ & 0.83786500 & -1.19857200 & 0.06276200 \\
\hline $\mathrm{C}$ & -2.02750200 & -2.08170000 & -0.97261600 & $\mathrm{H}$ & 0.36602100 & -2.18060100 & 0.12148000 \\
\hline $\mathrm{C}$ & -3.93759700 & -2.31303300 & 1.02914000 & $\mathrm{C}$ & 4.88553100 & -0.31585700 & 1.30986700 \\
\hline $\mathrm{H}$ & -3.14205300 & -0.46388600 & 1.78374500 & $\mathrm{H}$ & 4.76577200 & -1.39543500 & 1.39184200 \\
\hline $\mathrm{C}$ & -2.88822900 & -3.17472800 & -0.95616800 & $\mathrm{H}$ & 5.95127900 & -0.06636200 & 1.34031100 \\
\hline $\mathrm{H}$ & -1.28146600 & -1.99418400 & -1.75692200 & $\mathrm{H}$ & 4.39765000 & 0.16072600 & 2.16601400 \\
\hline $\mathrm{C}$ & -3.85005000 & -3.29494000 & 0.04479200 & $\mathrm{C}$ & 4.89403800 & -0.48022000 & -1.21791400 \\
\hline $\mathrm{H}$ & -4.68195600 & -2.39634200 & 1.81585700 & $\mathrm{H}$ & 5.96117800 & -0.24143800 & -1.27217500 \\
\hline $\mathrm{H}$ & -2.81257800 & -3.93273000 & -1.73041500 & $\mathrm{H}$ & 4.76853400 & -1.56101300 & -1.15980200 \\
\hline $\mathrm{H}$ & -4.52547700 & -4.14496800 & 0.05760500 & $\mathrm{H}$ & 4.41414300 & -0.11554400 & -2.13160700 \\
\hline $\mathrm{C}$ & -1.69424500 & 1.44670200 & -0.03901300 & $\mathrm{C}$ & 4.44208200 & 1.71087600 & -0.09062100 \\
\hline $\mathrm{C}$ & -3.05351700 & 1.72834500 & -0.27207300 & $\mathrm{H}$ & 3.97814000 & 2.09309500 & -1.00415200 \\
\hline $\mathrm{C}$ & -0.83587500 & 2.55084100 & 0.14390600 & $\mathrm{H}$ & 3.97399900 & 2.20339600 & 0.76663100 \\
\hline $\mathrm{C}$ & -3.52741500 & 3.03689900 & -0.31133700 & $\mathrm{H}$ & 5.50499200 & 1.97074700 & -0.10450800 \\
\hline $\mathrm{H}$ & -3.75026500 & 0.91157500 & -0.43234100 & & & & \\
\hline $\mathrm{C}$ & -1.31021500 & 3.85338500 & 0.09831200 & & & & \\
\hline $\mathrm{H}$ & 0.21539900 & 2.34747100 & 0.31784600 & & & & \\
\hline
\end{tabular}

DACN-2 $\mathrm{H}^{+}, \mathbf{1 - 2 \mathrm { H } ^ { + }}$ 
Sum of electronic and thermal Enthalpies=

Sum of electronic and thermal Free Energies=

21

$\mathrm{N}$

$\mathrm{H}$

C

$\mathrm{N}$

C

$\mathrm{N}$

C

$\mathrm{N}$

$\mathrm{H}$

C

$\mathrm{N}$

C

$\mathrm{N}$

C

C

$\mathrm{H}$

C

$\mathrm{H}$

C

C

$\mathrm{H}$

C

$\mathrm{H}$

$\mathrm{C}$

C

C

$\mathrm{H}$

C

$\mathrm{H}$

$\mathrm{H}$

$\mathrm{H}$

C

$\mathrm{H}$

$\mathrm{H}$

$\mathrm{H}$

C

$\mathrm{H}$

C

$\mathrm{H}$

$\mathrm{H}$

$\mathrm{H}$

C

$\mathrm{H}$

$\mathrm{H}$

$\mathrm{H}$

C

$\mathrm{H}$

C

$\mathrm{H}$

$\mathrm{H}$

$\mathrm{H}$
$-1889.350851$

$-1889.483124$

\section{C.}

$\begin{array}{lll}1.13027500 & 0.69788100 & -1.25314600\end{array}$

$\begin{array}{lll}0.17370300 & 0.40199600 & -1.40966000\end{array}$

$\begin{array}{llll}2.01249900 & -0.22835000 & -0.84830200\end{array}$

$\begin{array}{lll}4.39692500 & -0.45720300 & 0.26370900\end{array}$

$\begin{array}{llll}3.19580200 & -0.65537000 & -0.27317900\end{array}$

$\begin{array}{lll}1.75458900 & -2.80663400 & -0.81154400\end{array}$

$\begin{array}{lll}2.22853500 & -1.58011100 & -0.67789400\end{array}$

$\begin{array}{lll}-1.54176900 & 1.45842500 & -1.33242200\end{array}$

$\begin{array}{lll}-1.86696900 & 1.42311800 & -2.29359300\end{array}$

$\begin{array}{lll}-2.31684300 & 0.77123700 & -0.45325600\end{array}$

$\begin{array}{lll}-2.12505900 & 0.51304200 & 2.09834900\end{array}$

$\begin{array}{lll}-2.51785900 & 0.38845600 & 0.84743700\end{array}$

$\begin{array}{lll}-4.23318200 & -0.94994200 & -0.67410900\end{array}$

$\begin{array}{lll}-3.30064000 & -0.14991300 & -0.19270200\end{array}$

$\begin{array}{llll}1.39474900 & 2.07695800 & -1.38030900\end{array}$

$\begin{array}{llll}2.66109000 & 2.48775900 & -1.71627800\end{array}$

$\begin{array}{llll}3.39425600 & 1.74323900 & -2.00907400\end{array}$

$3.02436100 \quad 3.84903700 \quad-1.65016800$

$\begin{array}{llll}4.03851300 & 4.14096000 & -1.90169400\end{array}$

$\begin{array}{llll}2.11287200 & 4.78002300 & -1.23653100\end{array}$

$\begin{array}{llll}2.39083000 & 5.82406700 & -1.13360200\end{array}$

$\begin{array}{llll}0.77249300 & 4.40110800 & -0.95770800\end{array}$

$-0.17091900 \quad 5.37308000 \quad-0.53560300$

$\begin{array}{lll}0.16572400 & 6.39758500 & -0.40899800\end{array}$

$\begin{array}{llll}-1.47635900 & 5.03736800 & -0.29442800\end{array}$

$\begin{array}{lll}-2.19016700 & 5.78428200 & 0.03455100\end{array}$

$\begin{array}{lll}-1.90966100 & 3.71967300 & -0.54012100\end{array}$

$\begin{array}{llll}-2.96187800 & 3.46811200 & -0.44336400\end{array}$

$\begin{array}{lll}-1.02197600 & 2.75391500 & -0.94756600\end{array}$

$\begin{array}{llll}0.36812900 & 3.03924200 & -1.09874100\end{array}$

$\begin{array}{lll}5.21960000 & -1.66767600 & 0.50971900\end{array}$

$\begin{array}{llll}4.91846300 & -2.37744100 & -0.26477500\end{array}$

$\begin{array}{llll}6.70679800 & -1.40201100 & 0.29358100\end{array}$

$6.89187000 \quad-0.99874800 \quad-0.70546400$

$\begin{array}{lll}7.24720700 & -2.34809200 & 0.38827000\end{array}$

$\begin{array}{lll}7.11760600 & -0.71199100 & 1.03699100\end{array}$

$\begin{array}{lll}4.93225900 & -2.26791700 & 1.88735000\end{array}$

$\begin{array}{lll}3.85936300 & -2.42995200 & 2.03780900\end{array}$

$\begin{array}{lll}5.29079400 & -1.61412000 & 2.68883800\end{array}$

$\begin{array}{lll}5.44191100 & -3.23071900 & 1.98899600\end{array}$

$\begin{array}{lll}4.74412000 & 0.78249500 & 1.00685400\end{array}$

$\begin{array}{lll}5.37372000 & 0.43298400 & 1.82973500\end{array}$

$\begin{array}{lll}5.57342400 & 1.75880300 & 0.17641500\end{array}$

$\begin{array}{llll}6.46780900 & 1.28077300 & -0.22900000\end{array}$

$\begin{array}{llll}5.88945700 & 2.59357100 & 0.80975200\end{array}$

$\begin{array}{llll}4.99607500 & 2.16730400 & -0.65322400\end{array}$

$\begin{array}{lll}3.51971600 & 1.43112300 & 1.64431900\end{array}$

$\begin{array}{lll}2.96556500 & 0.69922800 & 2.23871000\end{array}$

$\begin{array}{lll}2.84583100 & 1.87208600 & 0.90446300\end{array}$

$\begin{array}{lll}3.84712100 & 2.23511000 & 2.30919400\end{array}$

$\begin{array}{lll}0.39176600 & -2.97989500 & -1.35718900\end{array}$

$\begin{array}{llll}0.23826000 & -4.05922400 & -1.41608200\end{array}$

$\begin{array}{lll}-0.64780400 & -2.40955400 & -0.39137100\end{array}$

$\begin{array}{lll}-0.61405800 & -2.92467900 & 0.57244000\end{array}$

$\begin{array}{lll}-1.65322500 & -2.52126800 & -0.80738200\end{array}$

$-0.47474100 \quad-1.34416500 \quad-0.20051000$ $\begin{array}{lll}0.28002600 & -2.42522800 & -2.77549900\end{array}$

$\begin{array}{lll}1.04864700 & -2.86360000 & -3.41801900\end{array}$

$\begin{array}{lll}0.39175700 & -1.33627300 & -2.81114700\end{array}$

$\begin{array}{lll}-0.69969000 & -2.67329300 & -3.19251700\end{array}$

$\begin{array}{lll}2.49613200 & -3.98181500 & -0.30885700\end{array}$

$\begin{array}{lll}3.42979900 & -3.58746000 & 0.09025500\end{array}$

$\begin{array}{lll}1.75430200 & -4.66169300 & 0.84021200\end{array}$

$\begin{array}{lll}1.54240400 & -3.94683400 & 1.64113500\end{array}$

$\begin{array}{llll}2.37649800 & -5.46319300 & 1.24828000\end{array}$

$\begin{array}{lll}0.81121200 & -5.10956400 & 0.50998700\end{array}$

$\begin{array}{lll}2.83625300 & -4.93717000 & -1.45024500\end{array}$

$\begin{array}{lll}3.37831700 & -4.41378400 & -2.24297400\end{array}$

$\begin{array}{lll}1.93877000 & -5.39094300 & -1.88325800\end{array}$

$3.46727500 \quad-5.74685700 \quad-1.07282600$

$\begin{array}{lll}-0.97612600 & 1.40629700 & 2.37791000\end{array}$

$\begin{array}{lll}-0.62412600 & 1.74617700 & 1.40090000\end{array}$

$\begin{array}{lll}0.16642000 & 0.64297500 & 3.04120000\end{array}$

$\begin{array}{lll}0.51682900 & -0.16966100 & 2.39578100\end{array}$

$\begin{array}{lll}-0.12355300 & 0.22295600 & 4.00935900\end{array}$

$\begin{array}{lll}0.99724700 & 1.33132500 & 3.21775600\end{array}$

$\begin{array}{lll}-1.42098400 & 2.63483000 & 3.16615400\end{array}$

$\begin{array}{lll}-2.22165000 & 3.15900100 & 2.63604000\end{array}$

$\begin{array}{lll}-0.57700300 & 3.32094300 & 3.28109500\end{array}$

$\begin{array}{lll}-1.77762100 & 2.37178300 & 4.16751500\end{array}$

$\begin{array}{lll}-2.80354800 & -0.18832400 & 3.20739300\end{array}$

$\begin{array}{lll}-2.32305000 & 0.18720000 & 4.11345000\end{array}$

$\begin{array}{lll}-2.54108900 & -1.69249000 & 3.13946500\end{array}$

$\begin{array}{lll}-1.46762900 & -1.89224600 & 3.20668400\end{array}$

$\begin{array}{lll}-2.90408400 & -2.13393700 & 2.20606000\end{array}$

$\begin{array}{lll}-3.04066700 & -2.19941100 & 3.96969000\end{array}$

$\begin{array}{lll}-4.27695100 & 0.20419100 & 3.28781300\end{array}$

$\begin{array}{lll}-4.83001100 & -0.06208700 & 2.38276700\end{array}$

$\begin{array}{lll}-4.37365000 & 1.28370000 & 3.43445100\end{array}$

$\begin{array}{lll}-4.75122000 & -0.30452700 & 4.13141300\end{array}$

$\begin{array}{lll}-5.02664100 & -1.83339300 & 0.20481700\end{array}$

$\begin{array}{lll}-4.59473100 & -1.71547000 & 1.19887400\end{array}$

$\begin{array}{lll}-6.48506300 & -1.38466100 & 0.26442200\end{array}$

$\begin{array}{lll}-6.55944300 & -0.33846000 & 0.57675900\end{array}$

$\begin{array}{lll}-7.02749700 & -2.00115000 & 0.98672400\end{array}$

$-6.98317500 \quad-1.49258000-0.70450600$

$\begin{array}{lll}-4.86591500 & -3.29668900 & -0.19978300\end{array}$

$\begin{array}{llll}-3.81256100 & -3.59258600 & -0.18120700\end{array}$

$\begin{array}{lll}-5.26797400 & -3.49049000 & -1.19935600\end{array}$

$\begin{array}{lll}-5.41373500 & -3.92907100 & 0.50443500\end{array}$

$\begin{array}{lll}-4.54985100 & -0.86794900 & -2.11974800\end{array}$

$\begin{array}{llll}-5.38912100 & -1.55055600 & -2.26653800\end{array}$

$\begin{array}{lll}-3.38537000 & -1.37301900 & -2.96855800\end{array}$

$\begin{array}{lll}-3.15914700 & -2.41679000 & -2.73198000\end{array}$

$\begin{array}{lll}-2.47520700 & -0.78265400 & -2.81229200\end{array}$

$\begin{array}{lll}-3.64211100 & -1.30943900 & -4.02946900\end{array}$

$\begin{array}{llll}-5.01634100 & 0.53357100 & -2.50771800\end{array}$

$\begin{array}{llll}-5.85912400 & 0.84767700 & -1.88569800\end{array}$

$\begin{array}{llll}-5.33596200 & 0.53808200 & -3.55326300\end{array}$

$\begin{array}{lll}-4.21894600 & 1.27760300 & -2.40339000\end{array}$ 
Catalyst-Enolate Complex, $\mathbf{1 - 2 \mathbf { H } ^ { + } \cdot \mathbf { 4 } ^ { - }}$

\# opt=calcfe freq=noraman wb97xd/6-31 g(d)/def2sv scrf=(iefpcm,solvent=dichloromethane $)$

$\begin{array}{lc}\text { Zero-point correction }= & 1.353526 \\ \text { Thermal correction to Energy }= & 1.426216 \\ \text { Thermal correction to Enthalpy }= & 1.427160 \\ \text { Thermal correction to Gibbs Free Energy= } & 1.245099 \\ \text { Sum of electronic and zero-point Energies }= & -2830.200290 \\ \text { Sum of electronic and thermal Energies }= & -2830.127599 \\ \text { Sum of electronic and thermal Enthalpies }= & -2830.126655 \\ \text { Sum of electronic and thermal Free Energies }= & -2830.308717\end{array}$

11

$\mathrm{N} \quad 1.16499700 \quad 0.78084300 \quad-1.64954000$

$\mathrm{H} \quad 0.17954500 \quad 1.02594500-1.45856900$

C $\quad 1.41365900-0.44431100-2.12514200$

$\mathrm{N} \quad 3.46837000-2.07025800-2.25757100$

C $\quad 2.26871100-1.51039100-2.32715500$

$\mathrm{N} \quad-0.08977400-2.28728000 \quad-3.19474200$

$\begin{array}{llll}\mathrm{C} & 0.92138600 & -1.60868800 & -2.68287900\end{array}$

$\mathrm{N} \quad 0.88284200 \quad 1.59525400 \quad 1.02981000$

$\mathrm{H} \quad-0.03520000 \quad 1.36524300 \quad 0.61437200$

$\begin{array}{llll}\mathrm{C} & 1.52942000 & 0.74620700 & 1.82138800\end{array}$

$\begin{array}{llll}\mathrm{N} & 3.88805600 & 0.76330900 & 2.92350400\end{array}$

$\begin{array}{llll}\mathrm{C} & 2.65589000 & 0.42035900 & 2.55027000\end{array}$

$\begin{array}{llll}\mathrm{N} & 0.97132100 & -1.61187300 & 2.74799800\end{array}$

$\begin{array}{llll}\mathrm{C} & 1.58399900 & -0.46559500 & 2.47996400\end{array}$

$\begin{array}{llll}\text { C } & 2.09586800 & 1.84673800 & -1.73395400\end{array}$

$\begin{array}{llll}\mathrm{C} & 2.85546800 & 1.95997200 & -2.87641700\end{array}$

$\begin{array}{llll}\mathrm{H} & 2.74474100 & 1.21230900 & -3.65657500\end{array}$

$\begin{array}{lllll}\text { C } & 3.72999200 & 3.04578600 & -3.07306100\end{array}$

$\begin{array}{lllll}\mathrm{H} & 4.31925400 & 3.09838400 & -3.98239600\end{array}$

$\begin{array}{lllll}\text { C } & 3.78335200 & 4.04877800 & -2.14457900\end{array}$

$\mathrm{H} \quad 4.41526900 \quad 4.91752200 \quad-2.30212800$

$\begin{array}{llll}\text { C } & 3.00897500 & 3.97790500 & -0.95757700\end{array}$

$\begin{array}{llll}\text { C } & 3.07310200 & 5.03691100 & -0.01256900\end{array}$

$\begin{array}{llll}\mathrm{H} & 3.69804800 & 5.89502700 & -0.24176300\end{array}$

$\begin{array}{llll}\text { C } & 2.36089200 & 4.98700200 & 1.15385300\end{array}$

$\begin{array}{llll}\mathrm{H} & 2.40186200 & 5.80374800 & 1.86636800\end{array}$

$\begin{array}{llll}\mathrm{C} & 1.59922400 & 3.83856000 & 1.45111600\end{array}$

$\begin{array}{llll}\mathrm{H} & 1.08242600 & 3.75367900 & 2.40143700\end{array}$

$\begin{array}{llll}\mathrm{C} & 1.53391700 & 2.78236000 & 0.57621700\end{array}$

$\begin{array}{llll}\text { C } & 2.18334100 & 2.83902100 & -0.70127200\end{array}$

$\begin{array}{llll}\text { C } & 3.64299200 & -3.42570500 & -2.82211000\end{array}$

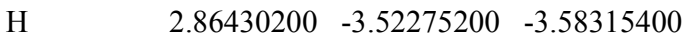

$\begin{array}{llll}\mathrm{C} & 4.98425900 & -3.57157800 & -3.53588500\end{array}$

$\mathrm{H} \quad \begin{array}{llll}\mathrm{C} & 5.10096800 & -2.80582400 & -4.30766600\end{array}$

$\mathrm{H} \quad 5.02872000 \quad-4.55362000 \quad-4.01515800$

$\mathrm{H} \quad \begin{array}{llll}5.82864900 & -3.50491500 & -2.84257700\end{array}$

C $\quad 3.42998100 \quad-4.49525900 \quad-1.74892700$

$\mathrm{H} \quad 2.46693300 \quad-4.36029600 \quad-1.24379300$

$\mathrm{H} \quad \begin{array}{llll}4.21841800 & -4.46030300 & -0.98973000\end{array}$

$\begin{array}{llll}\mathrm{H} & 3.44602900 & -5.49058800 & -2.20264800\end{array}$

C $\quad 4.49512100 \quad-1.53106400 \quad-1.33858300$
$-2830.308717$
$\begin{array}{lrrr}\mathrm{H} & 5.11392100 & -2.39130600 & -1.06943100 \\ \mathrm{C} & 5.38285200 & -0.48987600 & -2.01564500 \\ \mathrm{H} & 5.84296300 & -0.88892400 & -2.92315900 \\ \mathrm{H} & 6.18101200 & -0.18451200 & -1.33116500 \\ \mathrm{H} & 4.80355800 & 0.39969100 & -2.27965600 \\ \mathrm{C} & 3.86662800 & -0.99846400 & -0.05677200 \\ \mathrm{H} & 3.15297100 & -1.71634500 & 0.36147000 \\ \mathrm{H} & 3.35025600 & -0.04899700 & -0.22869100 \\ \mathrm{H} & 4.64604400 & -0.81726000 & 0.68617500 \\ \mathrm{C} & -1.31591000 & -1.56550700 & -3.60575800 \\ \mathrm{H} & -1.87005100 & -2.27544700 & -4.22493100 \\ \mathrm{C} & -2.16358400 & -1.19389100 & -2.39679600 \\ \mathrm{H} & -2.44569500 & -2.08617200 & -1.83155600 \\ \mathrm{H} & -3.07664800 & -0.67996800 & -2.71062700 \\ \mathrm{H} & -1.62282400 & -0.51830000 & -1.72925500 \\ \mathrm{C} & -0.97625300 & -0.35541900 & -4.47380000 \\ \mathrm{H} & -0.32752900 & -0.64043000 & -5.30778600 \\ \mathrm{H} & -0.48140300 & 0.43203600 & -3.89479100 \\ \mathrm{H} & -1.89818400 & 0.07031500 & -4.88007300 \\ \mathrm{C} & 0.02215700 & -3.74240900 & -3.41179200 \\ \mathrm{H} & 0.94986500 & -4.03922300 & -2.91850900 \\ \mathrm{C} & -1.11410600 & -4.49258100 & -2.71777700 \\ \mathrm{H} & -1.14861600 & -4.24564400 & -1.65210100 \\ \mathrm{H} & -0.94986600 & -5.56933500 & -2.81935400 \\ \mathrm{H} & -2.08766800 & -4.26317400 & -3.16254700 \\ \mathrm{C} & 0.13375000 & -4.07285900 & -4.89886900 \\ \mathrm{H} & 0.97200300 & -3.53592600 & -5.35398100 \\ \mathrm{H} & -0.77989600 & -3.80436400 & -5.43927000 \\ \mathrm{H} & 0.29603800 & -5.14661500 & -5.03098900 \\ \mathrm{C} & 4.44095000 & 2.04210800 & 2.42586400 \\ \mathrm{H} & 3.78950500 & 2.34053900 & 1.60338600 \\ \mathrm{C} & 5.84244300 & 1.85930300 & 1.84752400 \\ \mathrm{H} & 5.84319100 & 1.10384000 & 1.05668600 \\ \mathrm{H} & 6.57339900 & 1.56949400 & 2.60939600 \\ \mathrm{H} & 6.17587900 & 2.80653500 & 1.41336200 \\ \mathrm{C} & 4.38044400 & 3.12811400 & 3.49790000 \\ \mathrm{H} & 3.35839000 & 3.24520200 & 3.87110400 \\ \mathrm{H} & 4.70294100 & 4.08389900 & 3.07420000 \\ & 5.03545200 & 2.89633300 & 4.34495700 \\ \mathrm{H} & 4.60093000 & 0.02990000 & 3.98715300 \\ \mathrm{H} & 5.44443500 & 0.67106100 & 4.25477700\end{array}$

$\mathrm{C}$

$\mathrm{H}$

$\mathrm{H}$

$\mathrm{H}$

$\mathrm{C}$

$\mathrm{H}$

$\mathrm{H}$

$\mathrm{H}$

C

$\mathrm{H}$

C

$\mathrm{H}$

$\mathrm{H}$

$\mathrm{H}$

C

$\mathrm{H}$

$\mathrm{H}$

$\mathrm{H}$

C

H 


\begin{tabular}{|c|c|c|c|c|c|c|c|}
\hline $\mathrm{C}$ & 5.18329300 & -1.28531500 & 3.46814500 & $\mathrm{C}$ & -5.11775300 & -1.30337900 & 2.74551200 \\
\hline $\mathrm{H}$ & 5.93224600 & -1.08932600 & 2.69487200 & $\mathrm{C}$ & -4.35866800 & -2.14938400 & 0.62900600 \\
\hline $\mathrm{H}$ & 4.41731900 & -1.93347700 & 3.03223000 & $\mathrm{C}$ & -4.63996900 & -2.45042700 & 3.37594100 \\
\hline $\mathrm{H}$ & 5.66758700 & -1.83144900 & 4.28312200 & $\mathrm{H}$ & -5.60480100 & -0.52717100 & 3.32987100 \\
\hline $\mathrm{C}$ & 3.74392600 & -0.10856900 & 5.24548400 & $\mathrm{C}$ & -3.87219000 & -3.29345600 & 1.25483900 \\
\hline $\mathrm{H}$ & 2.83370900 & -0.68972800 & 5.07372000 & $\mathrm{H}$ & -4.25351700 & -2.02998100 & -0.44531700 \\
\hline $\mathrm{H}$ & 3.44594900 & 0.87931500 & 5.60910900 & $\mathrm{C}$ & -4.00922200 & -3.44676200 & 2.63386900 \\
\hline $\mathrm{H}$ & 4.31558400 & -0.60971700 & 6.03166300 & $\mathrm{H}$ & -4.75628300 & -2.56385800 & 4.44981600 \\
\hline $\mathrm{C}$ & 1.64104000 & -2.68713800 & 3.49621400 & $\mathrm{H}$ & -3.39054100 & -4.06834900 & 0.66413400 \\
\hline $\mathrm{H}$ & 2.61563800 & -2.28493100 & 3.77279000 & $\mathrm{H}$ & -3.63303600 & -4.33947600 & 3.12490200 \\
\hline $\mathrm{C}$ & 0.89250500 & -3.01825400 & 4.78668500 & $\mathrm{C}$ & -6.90688400 & 0.40595200 & 0.65674600 \\
\hline $\mathrm{H}$ & 0.74286200 & -2.11635100 & 5.38786400 & $\mathrm{C}$ & -7.85876200 & -0.52815800 & 1.09265200 \\
\hline $\mathrm{H}$ & 1.47396100 & -3.73484800 & 5.37421300 & $\mathrm{C}$ & -7.37537800 & 1.63906100 & 0.17062400 \\
\hline $\mathrm{H}$ & -0.08629500 & -3.46704000 & 4.58870600 & $\mathrm{C}$ & -9.22106100 & -0.24112700 & 1.04838700 \\
\hline $\mathrm{C}$ & 1.87612200 & -3.91353800 & 2.61445200 & $\mathrm{H}$ & -7.53287000 & -1.49429000 & 1.46613900 \\
\hline $\mathrm{H}$ & 2.45671800 & -3.64875800 & 1.72455700 & $\mathrm{C}$ & -8.73324700 & 1.92241400 & 0.12406200 \\
\hline $\mathrm{H}$ & 0.93260400 & -4.36477500 & 2.28979700 & $\mathrm{H}$ & -6.64634400 & 2.36562500 & -0.17230600 \\
\hline $\mathrm{H}$ & 2.43142500 & -4.67047900 & 3.17632300 & $\mathrm{C}$ & -9.66877800 & 0.98461400 & 0.56493100 \\
\hline $\mathrm{C}$ & -0.39721300 & -1.81453800 & 2.22737500 & $\mathrm{H}$ & -9.93551300 & -0.98481000 & 1.39101000 \\
\hline $\mathrm{H}$ & -0.69991100 & -2.80187300 & 2.58471000 & $\mathrm{H}$ & -9.06762700 & 2.88375600 & -0.25730300 \\
\hline $\mathrm{C}$ & -0.41898600 & -1.84637600 & 0.70001500 & $\mathrm{H}$ & -10.73116200 & 1.20768400 & 0.52947600 \\
\hline $\mathrm{H}$ & 0.29456500 & -2.58212300 & 0.31201800 & $\mathrm{O}$ & -3.06725200 & 2.67533400 & -1.31944200 \\
\hline $\mathrm{H}$ & -0.18417900 & -0.86689900 & 0.27136400 & $\mathrm{C}$ & -2.32906400 & 3.77838800 & -1.89534600 \\
\hline $\mathrm{H}$ & -1.42230400 & -2.11477200 & 0.35856300 & $\mathrm{C}$ & -1.57233900 & 4.55069500 & -0.81402300 \\
\hline $\mathrm{C}$ & -1.36135000 & -0.79128300 & 2.82003700 & $\mathrm{H}$ & -0.78808900 & 3.93574200 & -0.37110400 \\
\hline $\mathrm{H}$ & -1.29841800 & -0.79690600 & 3.91242000 & $\mathrm{H}$ & -1.11160200 & 5.44332000 & -1.25073000 \\
\hline $\mathrm{H}$ & -2.38657500 & -1.03226900 & 2.53160900 & $\mathrm{H}$ & -2.26246200 & 4.86985700 & -0.02595700 \\
\hline $\mathrm{H}$ & -1.14800300 & 0.22236700 & 2.46370800 & $\mathrm{C}$ & -1.39803700 & 3.30733600 & -3.01621700 \\
\hline $\mathrm{O}$ & -1.19990700 & 1.56662400 & -0.62314800 & $\mathrm{H}$ & -1.92958300 & 2.61559200 & -3.67782700 \\
\hline $\mathrm{C}$ & -2.47008400 & 1.68613700 & -0.61466800 & $\mathrm{H}$ & -1.07195200 & 4.16840900 & -3.60972700 \\
\hline $\mathrm{C}$ & -3.29968300 & 0.80753600 & 0.07126600 & $\mathrm{H}$ & -0.51308300 & 2.80846900 & -2.62084100 \\
\hline $\mathrm{H}$ & -2.79476100 & -0.02328200 & 0.55787400 & $\mathrm{C}$ & -3.43901900 & 4.64998300 & -2.48415000 \\
\hline $\mathrm{N}$ & -4.65124200 & 0.94779500 & 0.09521900 & $\mathrm{H}$ & -3.99151800 & 4.09952600 & -3.25235200 \\
\hline $\mathrm{C}$ & -5.45450700 & 0.11366900 & 0.67944100 & $\mathrm{H}$ & -4.14238700 & 4.95167300 & -1.70210200 \\
\hline $\mathrm{C}$ & -4.97988300 & -1.13271900 & 1.36344900 & $\mathrm{H}$ & -3.01342300 & 5.55013000 & -2.93867100 \\
\hline
\end{tabular}

Uncatalyzed Benzylation Transition State, TS1 $_{\mathbf{u}}$

\# opt=(calcfc,ts,noeigen) freq=noraman wb97xd/6-31g(d)/def2sv scrf=(iefpcm,solvent=dichloromethane $)$

Zero-point correction $=$

Thermal correction to Energy=

Thermal correction to Enthalpy=

Thermal correction to Gibbs Free Energy=

Sum of electronic and zero-point Energies=

Sum of electronic and thermal Energies=

Sum of electronic and thermal Enthalpies=

Sum of electronic and thermal Free Energies=
0.471798 (Hartree/Particle) 0.500100 0.501044

0.410493

$-3783.206422$

$-3783.178120$

$-3783.177175$

$-3783.267726$
$-11$

$\begin{array}{rrrr}\mathrm{O} & 2.42731500 & 0.77655000 & 2.43649100 \\ \mathrm{C} & 1.80738900 & -0.05169100 & 1.76334800 \\ \mathrm{C} & 0.39182200 & 0.03596300 & 1.51213900 \\ \mathrm{H} & -0.12886800 & 0.75342900 & 2.14586700 \\ \mathrm{~N} & -0.25475100 & -0.95865700 & 0.85512600 \\ \mathrm{C} & -1.53460000 & -1.01700900 & 0.65559400 \\ \mathrm{C} & -2.49975900 & -0.01854700 & 1.21519800 \\ \mathrm{C} & -3.24732400 & 0.81056500 & 0.37030200 \\ \mathrm{C} & -2.65214800 & 0.13184900 & 2.59834500\end{array}$

$\begin{array}{lrrr}\mathrm{C} & -4.11469100 & 1.76761300 & 0.88980700 \\ \mathrm{H} & -3.14957300 & 0.69829900 & -0.70650600 \\ \mathrm{C} & -3.52040100 & 1.08567100 & 3.12162700 \\ \mathrm{H} & -2.07917000 & -0.50621200 & 3.26571400 \\ \mathrm{C} & -4.25360600 & 1.90812200 & 2.26881200 \\ \mathrm{H} & -4.68183500 & 2.40438400 & 0.21717300 \\ \mathrm{H} & -3.62607700 & 1.18718000 & 4.19796400 \\ \mathrm{H} & -4.93018600 & 2.65368400 & 2.67605100 \\ \mathrm{C} & -2.05211800 & -2.11172100 & -0.19854400\end{array}$




$\begin{array}{lrrr}\mathrm{C} & -3.42330200 & -2.38748900 & -0.30181900 \\ \mathrm{C} & -1.16373400 & -2.91118400 & -0.93938200 \\ \mathrm{C} & -3.89066500 & -3.41419100 & -1.11954300 \\ \mathrm{H} & -4.13417400 & -1.79366500 & 0.26498400 \\ \mathrm{C} & -1.63085900 & -3.93458600 & -1.75162500 \\ \mathrm{H} & -0.10237700 & -2.69890200 & -0.86718300 \\ \mathrm{C} & -2.99986100 & -4.19310500 & -1.85139800 \\ \mathrm{H} & -4.95892500 & -3.60386200 & -1.18239300 \\ \mathrm{H} & -0.92361800 & -4.53324100 & -2.31995400 \\ \mathrm{H} & -3.36377600 & -4.99009200 & -2.49354500 \\ \mathrm{O} & 2.40339000 & -1.10083300 & 1.14512900 \\ \mathrm{C} & 3.78144900 & -1.45865300 & 1.38935800 \\ \mathrm{C} & 0.03060100 & 0.28761900 & -2.03712900 \\ \mathrm{C} & 0.49544300 & -0.55316400 & -3.04062400 \\ \mathrm{C} & 1.86498800 & -0.68282400 & -3.26447400 \\ \mathrm{C} & 2.76686700 & 0.04088200 & -2.48405000 \\ \mathrm{C} & 2.30204200 & 0.87498500 & -1.47571400 \\ \mathrm{C} & 0.92946500 & 0.99467300 & -1.23048400 \\ \mathrm{H} & -1.03571900 & 0.39168700 & -1.86384100 \\ \mathrm{H} & -0.21183700 & -1.11379700 & -3.64443100\end{array}$

Uncatalyzed Benzylation Transition State, $\mathbf{T S 1} 1_{\mathbf{u}}$ - IRC precomplex

\# opt=calcfc freq=noraman wb97xd/6-31g(d)/def2sv scrf=(iefpcm,solvent=dichloromethane $)$

$\begin{array}{lc}\text { Zero-point correction }= & 0.471712 \text { (Hartree/Particle) } \\ \text { Thermal correction to Energy= } & 0.500773 \\ \text { Thermal correction to Enthalpy= } & 0.501717 \\ \text { Thermal correction to Gibbs Free Energy= } & 0.407639 \\ \text { Sum of electronic and zero-point Energies }= & -3783.224171 \\ \text { Sum of electronic and thermal Energies }= & -3783.195110 \\ \text { Sum of electronic and thermal Enthalpies }= & -3783.194166 \\ \text { Sum of electronic and thermal Free Energies }= & -3783.288244\end{array}$

$-11$

$\begin{array}{lrrr}\mathrm{O} & -2.09305500 & -0.04994300 & -3.16590900 \\ \mathrm{C} & -1.61404900 & -0.69374100 & -2.22324400 \\ \mathrm{C} & -0.21920500 & -0.76533900 & -1.92810500 \\ \mathrm{H} & 0.43413700 & -0.21301600 & -2.60765200 \\ \mathrm{~N} & 0.22865700 & -1.39155000 & -0.83574800 \\ \mathrm{C} & 1.46244600 & -1.40053600 & -0.39723500 \\ \mathrm{C} & 2.56508800 & -0.68041200 & -1.10578400 \\ \mathrm{C} & 3.06570800 & 0.52683300 & -0.60412300 \\ \mathrm{C} & 3.08592700 & -1.16035600 & -2.31324600 \\ \mathrm{C} & 4.05398600 & 1.23418100 & -1.28405800 \\ \mathrm{H} & 2.65841200 & 0.92489500 & 0.32187000 \\ \mathrm{C} & 4.07722400 & -0.45971400 & -2.99561800 \\ \mathrm{H} & 2.70085300 & -2.09180800 & -2.72051100 \\ \mathrm{C} & 4.56666900 & 0.74006700 & -2.48156400 \\ \mathrm{H} & 4.41857600 & 2.17451700 & -0.87920800 \\ \mathrm{H} & 4.47037000 & -0.85133700 & -3.93004000 \\ \mathrm{H} & 5.33862100 & 1.28825400 & -3.01442600 \\ \mathrm{C} & 1.73385700 & -2.07903100 & 0.87776400 \\ \mathrm{C} & 3.03041600 & -2.17762500 & 1.41569100 \\ \mathrm{C} & 0.68411800 & -2.65988800 & 1.62169700 \\ \mathrm{C} & 3.26275300 & -2.80941300 & 2.63466900\end{array}$

$\begin{array}{lrrr}\mathrm{H} & 3.86796600 & -1.74965800 & 0.87205100 \\ \mathrm{C} & 0.92035400 & -3.28894500 & 2.83513800 \\ \mathrm{H} & -0.32194600 & -2.59675400 & 1.21830600 \\ \mathrm{C} & 2.21319700 & -3.37020000 & 3.35805200 \\ \mathrm{H} & 4.27782500 & -2.86454500 & 3.02089400 \\ \mathrm{H} & 0.08591400 & -3.72290900 & 3.38133600 \\ \mathrm{H} & 2.39584600 & -3.86112500 & 4.30963500 \\ \mathrm{O} & -2.39380700 & -1.41385100 & -1.35890000 \\ \mathrm{C} & -3.82334800 & -1.25203900 & -1.31149900 \\ \mathrm{C} & -1.08031300 & 0.50730100 & 1.51042800 \\ \mathrm{C} & -1.97400800 & 0.06156000 & 2.47970600 \\ \mathrm{C} & -3.03703800 & 0.86963300 & 2.87678700 \\ \mathrm{C} & -3.20240000 & 2.12992200 & 2.30253000 \\ \mathrm{C} & -2.30960800 & 2.57456400 & 1.33305300 \\ \mathrm{C} & -1.24642300 & 1.76345900 & 0.92617700 \\ \mathrm{H} & -0.26816300 & -0.13209800 & 1.18164000 \\ \mathrm{H} & -1.83572400 & -0.92129700 & 2.92150400 \\ \mathrm{H} & -3.73699500 & 0.51985900 & 3.63008200 \\ \mathrm{H} & -4.02990500 & 2.76376700 & 2.60750900 \\ \mathrm{H} & -2.43955600 & 3.55514200 & 0.88158700 \\ \mathrm{C} & -0.30214100 & 2.22107200 & -0.13720900\end{array}$




$\begin{array}{lrrrrrrr}\mathrm{H} & -0.78658200 & 2.82868600 & -0.89990300 & \mathrm{H} & -5.31272400 & -2.07319700 & 0.03590900 \\ \mathrm{H} & 0.23331800 & 1.39411500 & -0.60251400 & \mathrm{H} & -3.92570200 & -3.14669600 & -0.25647100 \\ \mathrm{Br} & 1.10216400 & 3.40665300 & 0.61554700 & \mathrm{H} & -3.74336800 & -1.72809500 & 0.80006300 \\ \mathrm{C} & -4.20262800 & 0.20776900 & -1.05334000 & \mathrm{C} & -4.47547200 & -1.79566500 & -2.58504500 \\ \mathrm{H} & -3.91741600 & 0.83683900 & -1.89762200 & \mathrm{H} & -4.16308800 & -2.83244100 & -2.75214000 \\ \mathrm{H} & -5.28443100 & 0.28864000 & -0.89711700 & \mathrm{H} & -5.56693500 & -1.77802500 & -2.48511100 \\ \mathrm{H} & -3.69583700 & 0.57074500 & -0.15332900 & \mathrm{H} & -4.18222700 & -1.19638400 & -3.44774000 \\ \mathrm{C} & -4.22722200 & -2.10474300 & -0.10729700 & & & & \end{array}$

Uncatalyzed Benzylation Transition State TS1 $\mathbf{u}_{\mathbf{u}}$ - IRC product

\# opt=calcfc freq=noraman wb97xd/6-31g(d)/def2sv scrf=(iefpcm,solvent=dichloromethane $)$

$\begin{array}{lc}\text { Zero-point correction= } & 0.475926 \text { (Hartree/Particle) } \\ \text { Thermal correction to Energy= } & 0.503449 \\ \text { Thermal correction to Enthalpy= } & 0.504393 \\ \text { Thermal correction to Gibbs Free Energy }= & 0.415128 \\ \text { Sum of electronic and zero-point Energies }= & -3783.296141 \\ \text { Sum of electronic and thermal Energies }= & -3783.268618 \\ \text { Sum of electronic and thermal Enthalpies }= & -3783.267674 \\ \text { Sum of electronic and thermal Free Energies }= & -3783.356939\end{array}$

$\begin{array}{crrr}-11 & & & \\ \mathrm{O} & 2.97421600 & 1.00903300 & -1.74094700 \\ \mathrm{C} & 2.14922800 & 0.86831500 & -0.85998400 \\ \mathrm{C} & 0.80464500 & 0.19018800 & -1.11216500 \\ \mathrm{H} & 0.28501800 & 0.86473400 & -1.80898500 \\ \mathrm{~N} & 0.06400300 & -0.04719100 & 0.10364600 \\ \mathrm{C} & -0.98401200 & 0.63010400 & 0.36065400 \\ \mathrm{C} & -1.45519800 & 1.80252500 & -0.44771400 \\ \mathrm{C} & -2.52531600 & 1.67087100 & -1.33537500 \\ \mathrm{C} & -0.81205500 & 3.03519400 & -0.31180400 \\ \mathrm{C} & -2.94452700 & 2.76972000 & -2.08075200 \\ \mathrm{H} & -2.99411000 & 0.69579600 & -1.45197400 \\ \mathrm{C} & -1.24158800 & 4.13386000 & -1.05133600 \\ \mathrm{H} & 0.02566900 & 3.12693700 & 0.37460700 \\ \mathrm{C} & -2.30918000 & 4.00207000 & -1.93682100 \\ \mathrm{H} & -3.76922200 & 2.66229700 & -2.77935100 \\ \mathrm{H} & -0.74118600 & 5.09108600 & -0.93796000 \\ \mathrm{H} & -2.64291100 & 4.85745800 & -2.51699800 \\ \mathrm{C} & -1.78667900 & 0.25559500 & 1.55854400 \\ \mathrm{C} & -2.71760200 & 1.13797100 & 2.11296500 \\ \mathrm{C} & -1.61428400 & -1.00542700 & 2.14148100 \\ \mathrm{C} & -3.45322500 & 0.77486200 & 3.23882700 \\ \mathrm{H} & -2.86785200 & 2.11586100 & 1.66592900 \\ \mathrm{C} & -2.34922100 & -1.36771600 & 3.26219800 \\ \mathrm{H} & -0.90140900 & -1.69011500 & 1.69482700 \\ \mathrm{C} & -3.27062800 & -0.47787300 & 3.81613100 \\ \mathrm{H} & -4.17101200 & 1.47212600 & 3.66096300 \\ \mathrm{H} & -2.21139100 & -2.35070400 & 3.70317900 \\ \mathrm{H} & -3.84741100 & -0.76501600 & 4.69072100 \\ \mathrm{O} & 2.26976600 & 1.30204300 & 0.38891900\end{array}$

$\mathbf{1 - 2} \mathbf{H}^{+}$catalyzed benzylation, TS1 
\# opt=(calcfc,ts,noeigen) freq=noraman wb97xd/6-31g(d)/def2sv scrf=(iefpcm,solvent=dichloromethane $)$

$\begin{array}{lc}\text { Zero-point correction= } & 1.474045 \text { (Hartree/Particle) } \\ \text { Thermal correction to Energy= } & 1.555729 \\ \text { Thermal correction to Enthalpy= } & 1.556673 \\ \text { Thermal correction to Gibbs Free Energy }= & 1.354817 \\ \text { Sum of electronic and zero-point Energies }= & -5672.698146 \\ \text { Sum of electronic and thermal Energies }= & -5672.616462 \\ \text { Sum of electronic and thermal Enthalpies }= & -5672.615518 \\ \text { Sum of electronic and thermal Free Energies }= & -5672.817374\end{array}$

11

C

$\mathrm{N}$

$\mathrm{C}$

$\mathrm{N}$

C

$\mathrm{N}$

$\mathrm{H}$

C

$\mathrm{N}$

C

$\mathrm{N}$

C

C

C

$\mathrm{H}$

C

$\mathrm{H}$

C

$\mathrm{H}$

$\mathrm{C}$

$\mathrm{C}$

$\mathrm{C}$

$\mathrm{H}$

C

$\mathrm{H}$

C

C

$\mathrm{C}$

C

$\mathrm{H}$

$\mathrm{H}$

$\mathrm{H}$

C

$\mathrm{H}$

$\mathrm{H}$

$\mathrm{H}$

C

$\mathrm{H}$

C

$\mathrm{H}$

$\mathrm{H}$

$\mathrm{H}$

$\begin{array}{rrr}-2.09483400 & -1.30141500 & 1.83910800 \\ -1.08983300 & -1.49831700 & 1.78672800 \\ -2.91382400 & -1.97845400 & 1.03575100 \\ -5.31342600 & -1.70121600 & 0.01915400 \\ -4.09850000 & -2.12415800 & 0.34058100 \\ -2.53593100 & -4.11345100 & -0.40369800 \\ -3.06272700 & -3.04488500 & 0.17340200 \\ -1.06552900 & 0.93429900 & 0.55118600 \\ -0.54515100 & 0.06011400 & 0.47895800 \\ -1.31809500 & 1.63630000 & -0.55967300 \\ -2.24040400 & 4.01780200 & -1.07576400 \\ -1.76682800 & 2.78465600 & -1.18120200 \\ -1.14238900 & 1.06895400 & -3.09317900 \\ -1.34472700 & 1.69259400 & -1.93326600 \\ -2.49827100 & -0.37597700 & 2.83870000 \\ -3.33815900 & -0.81091200 & 3.83612400 \\ -3.73629900 & -1.81986400 & 3.78388800 \\ -3.63769500 & 0.01072600 & 4.94073500 \\ -4.31445400 & -0.34910800 & 5.70821700 \\ -3.01426800 & 1.22307800 & 5.07049700 \\ -3.17991600 & 1.83704300 & 5.95070300 \\ -2.12927200 & 1.69835500 & 4.06847500 \\ -1.44686000 & 2.93259500 & 4.24250700 \\ -1.60891700 & 3.48919700 & 5.16071100 \\ -0.58543700 & 3.39653600 & 3.28698100 \\ -0.03736300 & 4.32118400 & 3.43328700 \\ -0.43634700 & 2.68847100 & 2.07502400 \\ 0.20811000 & 3.07298800 & 1.28694600 \\ -1.12085400 & 1.51924700 & 1.85108300 \\ -1.91974300 & 0.93013500 & 2.88419000 \\ -6.16932800 & -2.56916700 & -0.81641100 \\ -5.74905500 & -3.57294800 & -0.71237600 \\ -7.59876600 & -2.63142300 & -0.28262800 \\ -7.60979000 & -2.94537700 & 0.76474000 \\ -8.16666900 & -3.36015500 & -0.86800400 \\ -8.11377400 & -1.66925500 & -0.36658700 \\ -6.10486300 & -2.15496400 & -2.28599900 \\ -5.07048400 & -2.15252400 & -2.64535800 \\ -6.52070700 & -1.15281600 & -2.43597600 \\ -6.68307700 & -2.85251800 & -2.89918300 \\ -5.72193600 & -0.30299800 & 0.28469200 \\ -6.56045800 & -0.12534800 & -0.39323100 \\ -6.21666700 & -0.12190300 & 1.71733900 \\ -7.02345000 & -0.82385800 & 1.94479500 \\ -6.59463700 & 0.89596900 & 1.85478500 \\ -5.40563600 & -0.28010400 & 2.43368200\end{array}$

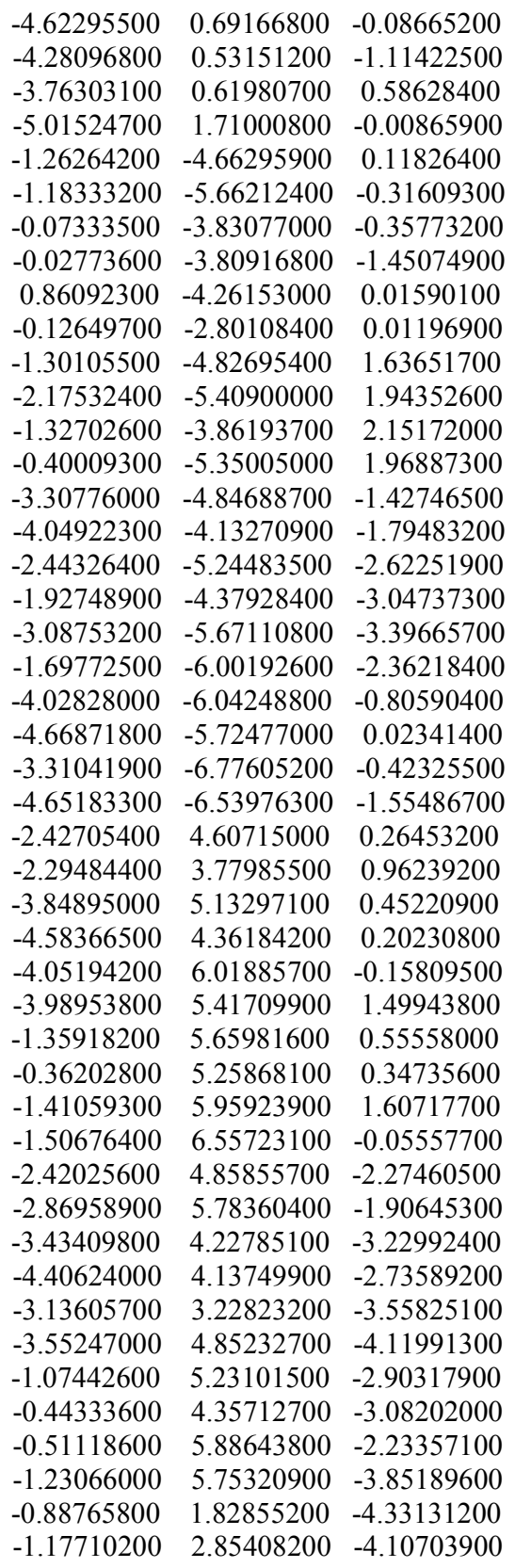




$\begin{array}{lrrr}\mathrm{C} & 0.60399800 & 1.83188600 & -4.66966800 \\ \mathrm{H} & 1.17870500 & 2.20235800 & -3.81420900 \\ \mathrm{H} & 0.78828300 & 2.48309200 & -5.52977500 \\ \mathrm{H} & 0.96121500 & 0.82768700 & -4.92523400 \\ \mathrm{C} & -1.76473500 & 1.34374400 & -5.48323200 \\ \mathrm{H} & -2.81996700 & 1.33187800 & -5.19279500 \\ \mathrm{H} & -1.48728900 & 0.33991600 & -5.82093000 \\ \mathrm{H} & -1.64724900 & 2.02020200 & -6.33501400 \\ \mathrm{C} & -0.85363200 & -0.37590500 & -3.06251800 \\ \mathrm{H} & -0.71293000 & -0.66612400 & -4.10581300 \\ \mathrm{C} & -2.05515200 & -1.13838600 & -2.51070700 \\ \mathrm{H} & -2.95882900 & -0.91490900 & -3.08584600 \\ \mathrm{H} & -2.23582700 & -0.86971700 & -1.46388100 \\ \mathrm{H} & -1.86910100 & -2.21530500 & -2.54623100 \\ \mathrm{C} & 0.42469700 & -0.69906900 & -2.30376100 \\ \mathrm{H} & 1.27022500 & -0.11490500 & -2.67380100 \\ \mathrm{H} & 0.67108500 & -1.75712700 & -2.41718000 \\ \mathrm{H} & 0.30988100 & -0.50395600 & -1.23548700 \\ \mathrm{O} & 0.59382900 & -1.08769000 & 1.51980600 \\ \mathrm{C} & 1.84501000 & -1.22429500 & 1.56259100 \\ \mathrm{C} & 2.64064500 & -1.22299200 & 0.38965100 \\ \mathrm{H} & 2.09153500 & -1.38838000 & -0.53160100 \\ \mathrm{~N} & 3.99423400 & -1.40293000 & 0.45141200 \\ \mathrm{C} & 4.72067300 & -1.54947400 & -0.60663500 \\ \mathrm{C} & 4.13721600 & -1.65659800 & -1.98574300 \\ \mathrm{C} & 4.22940100 & -0.60183300 & -2.89914600 \\ \mathrm{C} & 3.46594800 & -2.82176400 & -2.37233900 \\ \mathrm{C} & 3.65667000 & -0.70475500 & -4.16542400 \\ \mathrm{H} & 4.76201300 & 0.30412300 & -2.62200600 \\ \mathrm{C} & 2.90124500 & -2.93118600 & -3.63980500 \\ \mathrm{H} & 3.38721400 & -3.64514500 & -1.66747500 \\ \mathrm{C} & 2.99160800 & -1.87019200 & -4.53901800 \\ \mathrm{H} & 3.73359000 & 0.12542700 & -4.86139800 \\ \mathrm{H} & 2.38827000 & -3.84531600 & -3.92474700 \\ \mathrm{H} & 2.54884200 & -1.95307300 & -5.52716400 \\ \mathrm{C} & 6.19228000 & -1.62577900 & -0.44926600 \\ \mathrm{C} & 7.02874600 & -1.95982900 & -1.52201500 \\ \mathrm{C} & 6.78105400 & -1.35959200 & 0.79746700\end{array}$

$\begin{array}{rrr}8.41012900 & -2.02817500 & -1.35485100 \\ 6.59787300 & -2.17124700 & -2.49606100 \\ 8.15699700 & -1.42893800 & 0.96214700 \\ 6.13329400 & -1.09031600 & 1.62477700 \\ 8.98149200 & -1.76408500 & -0.11401200 \\ 9.04007100 & -2.29197800 & -2.19979700 \\ 8.59350900 & -1.21764700 & 1.93444500 \\ 10.05834000 & -1.81746200 & 0.01669400 \\ 2.51521300 & -1.32356400 & 2.71710300 \\ 1.87274200 & -1.42847100 & 4.01630500 \\ 4.81040200 & 1.67973900 & 0.43875800 \\ 5.81411900 & 2.00749300 & 1.34136300 \\ 5.49581900 & 2.27417400 & 2.67179700 \\ 4.16736500 & 2.21894800 & 3.09666500 \\ 3.16552300 & 1.88801300 & 2.19591500 \\ 3.47774400 & 1.60480400 & 0.86050600 \\ 5.05658300 & 1.47240400 & -0.59769200 \\ 6.84659600 & 2.04704400 & 1.00876400 \\ 6.28055800 & 2.52869200 & 3.37775500 \\ 3.91813000 & 2.43021000 & 4.13248900 \\ 2.13035000 & 1.83469200 & 2.51959800 \\ 2.43537800 & 1.18575400 & -0.05957100 \\ 1.43317600 & 1.01938000 & 0.29687700 \\ 2.69585800 & 0.83316800 & -1.04489100 \\ 1.66243400 & 3.27541800 & -1.06296200 \\ 1.19474100 & -0.11493600 & 4.39902500 \\ 0.45915400 & 0.17465500 & 3.64893600 \\ 0.68385200 & -0.23248800 & 5.36051900 \\ 1.93517000 & 0.68351100 & 4.50058700 \\ 0.89330600 & -2.60390600 & 4.05666300 \\ 0.67435200 & -2.85277700 & 5.10006100 \\ -0.04815600 & -2.36734200 & 3.55963200 \\ 1.33503000 & -3.48430600 & 3.57904000 \\ 3.05556500 & -1.70177900 & 4.94357500 \\ 3.52614600 & -2.65889700 & 4.69867900 \\ 3.80383500 & -0.90994500 & 4.84061000 \\ 2.71838700 & -1.73335700 & 5.98437100\end{array}$

$\mathbf{1 - 2 H ^ { + }}$ catalyzed TS1 - IRC precomplex

\begin{tabular}{|c|c|c|c|c|c|c|c|}
\hline \multicolumn{3}{|c|}{ Zero-point correction $=$} & \multicolumn{2}{|c|}{1.475825 (Hartree/Particle) } & & & \\
\hline \multicolumn{3}{|c|}{ Thermal correction to Energy $=$} & \multicolumn{2}{|c|}{1.557387} & & & \\
\hline \multicolumn{3}{|c|}{ Thermal correction to Enthalpy= } & & & & \\
\hline \multicolumn{3}{|c|}{ Thermal correction to Gibbs Free Energy= 1.356719} & \multicolumn{2}{|c|}{$\begin{array}{cc} & 1.558331 \\
\text { ergy }= & 1.356719\end{array}$} & & & \\
\hline \multicolumn{4}{|c|}{ Sum of electronic and zero-point Energies $=$} & -5672.715369 & & & \\
\hline \multicolumn{4}{|c|}{ Sum of electronic and thermal Energies $=$} & -5672.633807 & & & \\
\hline \multicolumn{4}{|c|}{ Sum of electronic and thermal Enthalpies $=$} & -5672.632863 & & & \\
\hline \multicolumn{4}{|c|}{ Sum of electronic and thermal Free Energies $=$} & -5672.834475 & & & \\
\hline \multicolumn{8}{|c|}{11} \\
\hline $\mathrm{N}$ & -2.22911400 & -1.55766200 & 1.43945200 & $\mathrm{~N}$ & -3.20161700 & -3.71976100 & -1.31039000 \\
\hline $\mathrm{H}$ & -1.26805200 & -1.89526100 & 1.28415000 & $\mathrm{C}$ & -3.52079400 & -2.73452800 & -0.48789600 \\
\hline $\mathrm{C}$ & -3.17067100 & -1.91610200 & 0.56848500 & $\mathrm{~N}$ & -0.69819900 & 0.65235600 & 0.76619500 \\
\hline $\mathrm{N}$ & -5.51948000 & -1.05138900 & -0.21077000 & $\mathrm{H}$ & -0.26231400 & -0.24165700 & 0.49136700 \\
\hline $\mathrm{C}$ & -4.38283600 & -1.72236600 & -0.06407200 & $\mathrm{C}$ & -1.01307000 & 1.59202800 & -0.12262800 \\
\hline
\end{tabular}




\begin{tabular}{|c|c|c|c|c|c|c|c|}
\hline $\mathrm{N}$ & -1.92222400 & 4.02381500 & 0.08865100 & $\mathrm{H}$ & -1.84599900 & 3.22921600 & 1.98210300 \\
\hline $\mathrm{C}$ & -1.48096600 & 2.86654600 & -0.38700300 & $\mathrm{C}$ & -3.40989200 & 4.66288600 & 1.98030000 \\
\hline $\mathrm{N}$ & -1.05289700 & 1.71521500 & -2.72742600 & $\mathrm{H}$ & -4.17403400 & 3.97553800 & 1.60607500 \\
\hline $\mathrm{C}$ & -1.14345300 & 2.00951600 & -1.42919900 & $\mathrm{H}$ & -3.65073200 & 5.67479900 & 1.63975600 \\
\hline $\mathrm{C}$ & -2.47107000 & -0.86827300 & 2.65569000 & $\mathrm{H}$ & -3.46012400 & 4.66496900 & 3.07330800 \\
\hline $\mathrm{C}$ & -3.41264300 & -1.36947800 & 3.52410200 & $\mathrm{C}$ & -0.91688500 & 5.15419500 & 2.05252000 \\
\hline $\mathrm{H}$ & -3.99192300 & -2.23870500 & 3.22657400 & $\mathrm{H}$ & 0.06366800 & 4.81581800 & 1.70352800 \\
\hline $\mathrm{C}$ & -3.59489700 & -0.80677800 & 4.80317000 & $\mathrm{H}$ & -0.91261100 & 5.17108800 & 3.14660100 \\
\hline $\mathrm{H}$ & -4.35260500 & -1.21429300 & 5.46385000 & $\mathrm{H}$ & -1.07673500 & 6.17925500 & 1.69999700 \\
\hline $\mathrm{C}$ & -2.77068600 & 0.20168100 & 5.22489100 & $\mathrm{C}$ & -2.19721400 & 5.15422200 & -0.81767500 \\
\hline $\mathrm{H}$ & -2.86002600 & 0.60532800 & 6.22894200 & $\mathrm{H}$ & -2.61641700 & 5.93482200 & -0.17914600 \\
\hline $\mathrm{C}$ & -1.77870200 & 0.73667800 & 4.36329100 & $\mathrm{C}$ & -3.27751400 & 4.78297600 & -1.83376300 \\
\hline $\mathrm{C}$ & -0.90469200 & 1.75590400 & 4.82881300 & $\mathrm{H}$ & -4.21452900 & 4.55524600 & -1.31678900 \\
\hline $\mathrm{H}$ & -1.00254200 & 2.09187600 & 5.85684700 & $\mathrm{H}$ & -3.00737900 & 3.90677200 & -2.43009400 \\
\hline $\mathrm{C}$ & 0.04777300 & 2.29351600 & 4.00760300 & $\mathrm{H}$ & -3.45374700 & 5.61581200 & -2.52039500 \\
\hline $\mathrm{H}$ & 0.73049500 & 3.05514400 & 4.36835800 & $\mathrm{C}$ & -0.90673600 & 5.71746100 & -1.41854900 \\
\hline $\mathrm{C}$ & 0.11636000 & 1.88212300 & 2.66083200 & $\mathrm{H}$ & -0.32832500 & 4.95710600 & -1.94962200 \\
\hline $\mathrm{H}$ & 0.81811300 & 2.35540500 & 1.98114000 & $\mathrm{H}$ & -0.26442700 & 6.11924600 & -0.62951100 \\
\hline $\mathrm{C}$ & -0.73169900 & 0.92157700 & 2.16731400 & $\mathrm{H}$ & -1.13788000 & 6.52431000 & -2.12003300 \\
\hline $\mathrm{C}$ & -1.66101200 & 0.25165400 & 3.02554400 & $\mathrm{C}$ & -0.83561500 & 2.77539400 & -3.72853800 \\
\hline $\mathrm{C}$ & -6.52724600 & -1.56761900 & -1.16034700 & $\mathrm{H}$ & -1.08803300 & 3.70714000 & -3.22371200 \\
\hline $\mathrm{H}$ & -6.31963300 & -2.63756400 & -1.24956100 & $\mathrm{C}$ & 0.63956200 & 2.84458500 & -4.12782200 \\
\hline $\mathrm{C}$ & -7.94129700 & -1.43863100 & -0.59835100 & $\mathrm{H}$ & 1.25851500 & 3.00045700 & -3.23830600 \\
\hline $\mathrm{H}$ & -8.01757200 & -1.92712500 & 0.37691900 & $\mathrm{H}$ & 0.80289300 & 3.67638700 & -4.82008100 \\
\hline $\mathrm{H}$ & -8.64320800 & -1.92272700 & -1.28340300 & $\mathrm{H}$ & 0.96696500 & 1.92367800 & -4.62318200 \\
\hline $\mathrm{H}$ & -8.25195000 & -0.39441700 & -0.49284800 & $\mathrm{C}$ & -1.77779100 & 2.63507200 & -4.92166800 \\
\hline $\mathrm{C}$ & -6.37570100 & -0.91443700 & -2.53445500 & $\mathrm{H}$ & -2.81654300 & 2.54557400 & -4.58888900 \\
\hline $\mathrm{H}$ & -5.36238600 & -1.05455800 & -2.92637700 & $\mathrm{H}$ & -1.53564600 & 1.76647600 & -5.54220700 \\
\hline $\mathrm{H}$ & -6.57572200 & 0.16126500 & -2.48607800 & $\mathrm{H}$ & -1.69199100 & 3.52309600 & -5.55463500 \\
\hline $\mathrm{H}$ & -7.08330600 & -1.35593700 & -3.24251100 & $\mathrm{C}$ & -0.86788700 & 0.30403000 & -3.11299000 \\
\hline $\mathrm{C}$ & -5.63595000 & 0.33660300 & 0.29320900 & $\mathrm{H}$ & -0.77585400 & 0.31022400 & -4.20089100 \\
\hline $\mathrm{H}$ & -6.43765100 & 0.78302400 & -0.30005900 & $\mathrm{C}$ & -2.10809400 & -0.50262900 & -2.73770000 \\
\hline $\mathrm{C}$ & -6.04806700 & 0.37616400 & 1.76173900 & $\mathrm{H}$ & -3.00758500 & -0.07573900 & -3.19190700 \\
\hline $\mathrm{H}$ & -6.98325800 & -0.16753200 & 1.92100000 & $\mathrm{H}$ & -2.23926300 & -0.51508900 & -1.64984700 \\
\hline $\mathrm{H}$ & -6.19294200 & 1.41410400 & 2.07837900 & $\mathrm{H}$ & -2.00085100 & -1.53892200 & -3.06820000 \\
\hline $\mathrm{H}$ & -5.27457700 & -0.06572300 & 2.39578500 & $\mathrm{C}$ & 0.40005200 & -0.30456600 & -2.53179700 \\
\hline $\mathrm{C}$ & -4.36795300 & 1.14374300 & 0.02028600 & $\mathrm{H}$ & 1.28388800 & 0.29670900 & -2.76252900 \\
\hline $\mathrm{H}$ & -4.06852700 & 1.06232200 & -1.02957600 & $\mathrm{H}$ & 0.55886900 & -1.30469100 & -2.94349700 \\
\hline $\mathrm{H}$ & -3.53616400 & 0.81506300 & 0.65213400 & $\mathrm{H}$ & 0.32261600 & -0.41644200 & -1.44843200 \\
\hline $\mathrm{H}$ & -4.55062700 & 2.19937500 & 0.23956400 & $\mathrm{O}$ & 0.41372600 & -1.79722900 & 0.99204200 \\
\hline $\mathrm{C}$ & -2.01319000 & -4.55566200 & -1.01798900 & $\mathrm{C}$ & 1.64548200 & -2.12870800 & 0.98432700 \\
\hline $\mathrm{H}$ & -2.12014500 & -5.43382600 & -1.65906200 & $\mathrm{C}$ & 2.50079200 & -1.85231600 & -0.07775800 \\
\hline $\mathrm{C}$ & -0.72491400 & -3.82879000 & -1.39873600 & $\mathrm{H}$ & 2.04580000 & -1.42278500 & -0.96459900 \\
\hline $\mathrm{H}$ & -0.73190400 & -3.55807200 & -2.45904500 & $\mathrm{~N}$ & 3.84773200 & -2.00917500 & 0.02584500 \\
\hline $\mathrm{H}$ & 0.13718300 & -4.47823400 & -1.21715500 & $\mathrm{C}$ & 4.68494500 & -1.69384000 & -0.91118500 \\
\hline $\mathrm{H}$ & -0.57732900 & -2.92407000 & -0.79963200 & $\mathrm{C}$ & 4.26175700 & -1.21233000 & -2.26550600 \\
\hline $\mathrm{C}$ & -2.01313200 & -5.04301900 & 0.42946000 & $\mathrm{C}$ & 4.58569300 & 0.07650900 & -2.70600600 \\
\hline $\mathrm{H}$ & -2.96136700 & -5.53058600 & 0.67579600 & $\mathrm{C}$ & 3.51591200 & -2.03491600 & -3.11706300 \\
\hline $\mathrm{H}$ & -1.84452800 & -4.22533800 & 1.13657400 & $\mathrm{C}$ & 4.15343900 & 0.54176600 & -3.94585400 \\
\hline $\mathrm{H}$ & -1.20465800 & -5.76633100 & 0.56873900 & $\mathrm{H}$ & 5.18573800 & 0.71602300 & -2.06367500 \\
\hline $\mathrm{C}$ & -4.12496900 & -4.09108900 & -2.40118800 & $\mathrm{C}$ & 3.09711000 & -1.58132300 & -4.36486100 \\
\hline $\mathrm{H}$ & -4.78478000 & -3.22976200 & -2.52662100 & $\mathrm{H}$ & 3.25921400 & -3.03882100 & -2.78979200 \\
\hline $\mathrm{C}$ & -3.39478200 & -4.28986600 & -3.72761600 & $\mathrm{C}$ & 3.40598900 & -0.28759100 & -4.78014400 \\
\hline $\mathrm{H}$ & -2.81752900 & -3.40142600 & -3.99795100 & $\mathrm{H}$ & 4.40471000 & 1.55021800 & -4.26231200 \\
\hline $\mathrm{H}$ & -4.13321900 & -4.46934200 & -4.51433100 & $\mathrm{H}$ & 2.52084900 & -2.23702100 & -5.01160000 \\
\hline $\mathrm{H}$ & -2.72036000 & -5.15131600 & -3.70613500 & $\mathrm{H}$ & 3.07226600 & 0.07119900 & -5.74960100 \\
\hline $\mathrm{C}$ & -4.96649800 & -5.30442200 & -2.00790100 & $\mathrm{C}$ & 6.13251200 & -1.77282900 & -0.60590000 \\
\hline $\mathrm{H}$ & -5.50297400 & -5.11788700 & -1.07190500 & $\mathrm{C}$ & 7.11020800 & -1.71211600 & -1.60951300 \\
\hline $\mathrm{H}$ & -4.34032800 & -6.19296000 & -1.87350100 & $\mathrm{C}$ & 6.56467900 & -1.90622300 & 0.72427500 \\
\hline $\mathrm{H}$ & -5.69901500 & -5.52423900 & -2.79013100 & $\mathrm{C}$ & 8.46594100 & -1.78075700 & -1.29701400 \\
\hline $\mathrm{C}$ & -2.01305200 & 4.21654300 & 1.55145900 & $\mathrm{H}$ & 6.80955300 & -1.61413000 & -2.64862100 \\
\hline
\end{tabular}




$\begin{array}{lrrr}\mathrm{C} & 7.91554700 & -1.97758800 & 1.03437700 \\ \mathrm{H} & 5.81321400 & -1.94235000 & 1.50535200 \\ \mathrm{C} & 8.87836100 & -1.91331000 & 0.02582800 \\ \mathrm{H} & 9.20266300 & -1.73423800 & -2.09449300 \\ \mathrm{H} & 8.22235700 & -2.07637600 & 2.07248400 \\ \mathrm{H} & 9.93566800 & -1.96535400 & 0.26929500 \\ \mathrm{O} & 2.19171700 & -2.80584900 & 2.02279900 \\ \mathrm{C} & 1.57269200 & -2.93527800 & 3.32059300 \\ \mathrm{C} & 4.97220400 & 2.04780200 & 0.45812400 \\ \mathrm{C} & 6.01365800 & 1.91473900 & 1.36837200 \\ \mathrm{C} & 5.80827500 & 1.23301000 & 2.56723200 \\ \mathrm{C} & 4.55961200 & 0.68659300 & 2.84934000 \\ \mathrm{C} & 3.51933300 & 0.81409600 & 1.93386200 \\ \mathrm{C} & 3.72029200 & 1.48982800 & 0.73079100 \\ \mathrm{H} & 5.12469300 & 2.59051700 & -0.47180500 \\ \mathrm{H} & 6.98625900 & 2.34166400 & 1.14445500 \\ \mathrm{H} & 6.62215100 & 1.12581000 & 3.27782500 \\ \mathrm{H} & 4.39772300 & 0.15306500 & 3.78134000\end{array}$

$\begin{array}{lrrr}\mathrm{H} & 2.55167200 & 0.36743400 & 2.14155600 \\ \mathrm{C} & 2.61326400 & 1.58800800 & -0.26411800 \\ \mathrm{H} & 1.76847500 & 0.95499800 & -0.00164100 \\ \mathrm{H} & 2.93468600 & 1.37128400 & -1.28143300 \\ \mathrm{Br} & 1.88891100 & 3.43582200 & -0.37189700 \\ \mathrm{C} & 1.36163000 & -1.56346000 & 3.95837300 \\ \mathrm{H} & 0.71042300 & -0.94645200 & 3.33890200 \\ \mathrm{H} & 0.89687900 & -1.67434600 & 4.94388100 \\ \mathrm{H} & 2.32051200 & -1.05143000 & 4.08719700 \\ \mathrm{C} & 0.26920600 & -3.73703000 & 3.25770900 \\ \mathrm{H} & 0.02381800 & -4.11648900 & 4.25539900 \\ \mathrm{H} & -0.56760000 & -3.12964000 & 2.91231200 \\ \mathrm{H} & 0.38628000 & -4.59123500 & 2.58357900 \\ \mathrm{C} & 2.62073200 & -3.72457000 & 4.10625400 \\ \mathrm{H} & 2.76735300 & -4.71374500 & 3.66089500 \\ \mathrm{H} & 3.57813900 & -3.19493900 & 4.09460000 \\ \mathrm{H} & 2.30163800 & -3.85272500 & 5.14540700\end{array}$

$1-2 \mathrm{H}^{+}$catalyzed TS1 - IRC product

\# opt=calcfc freq=noraman wb97xd/6-31g(d)/def2sv scrf=(iefpcm,solvent=dichloromethane $)$

$\begin{array}{lc}\text { Zero-point correction= } & 1.480009 \text { (Hartree/Particle) } \\ \text { Thermal correction to Energy= } & 1.561225 \\ \text { Thermal correction to Enthalpy= } & 1.562169 \\ \text { Thermal correction to Gibbs Free Energy }= & 1.360549 \\ \text { Sum of electronic and zero-point Energies }= & -5672.775055 \\ \text { Sum of electronic and thermal Energies }= & -5672.693839 \\ \text { Sum of electronic and thermal Enthalpies }= & -5672.692895 \\ \text { Sum of electronic and thermal Free Energies }= & -5672.894514\end{array}$

11

$\mathrm{N} \quad-1.19889700 \quad 1.03192800 \quad-2.16053000$

$\mathrm{H} \quad-0.22650600 \quad 0.84543400-1.92323500$

$\begin{array}{llll}\mathrm{C} & -1.73514800 & 2.17360500 & -1.72834200\end{array}$

$\mathrm{N} \quad-4.10787100 \quad 3.14257400-1.17734900$

C $\quad-2.80721100 \quad 2.95424500-1.34008600$

$\begin{array}{llll}\mathrm{N} & -0.62628600 & 4.35557200 & -0.85395700\end{array}$

$\begin{array}{llll}\mathrm{C} & -1.50171400 & 3.43176400 & -1.21488500\end{array}$

$\mathrm{N} \quad-1.22908900 \quad-0.98260600 \quad-0.27086900$

$\mathrm{H} \quad-0.31904500 \quad-0.53794500-0.19164400$

$\begin{array}{llll}\mathrm{C} & -1.97574000 & -1.09131800 & 0.82953300\end{array}$

$\begin{array}{llll}\mathrm{N} & -4.13773100 & -2.38754200 & 1.46068100\end{array}$

$\begin{array}{llll}\mathrm{C} & -3.07023500 & -1.59560800 & 1.49121300\end{array}$

$\begin{array}{llll}\mathrm{N} & -1.84123300 & 0.05603900 & 3.15066000\end{array}$

$\begin{array}{llll}\text { C } & -2.21856300 & -0.69895800 & 2.12271800\end{array}$

$\begin{array}{llll}\text { C } & -1.83437800 & 0.08132900 & -3.00386200\end{array}$

$\begin{array}{lllll}\text { C } & -2.34291400 & 0.50770200 & -4.20781900\end{array}$

$\mathrm{H} \quad-2.31453700 \quad 1.56733100 \quad-4.44530600$

$\begin{array}{llll}\text { C } & -2.84408300 & -0.41523800 & -5.14792700\end{array}$

$\mathrm{H} \quad-3.25298500 \quad-0.05429800 \quad-6.08554900$

$\begin{array}{llll}\text { C } & -2.74557700 & -1.75805400 & -4.89825000\end{array}$

$\mathrm{H} \quad-3.06909400 \quad-2.48228200 \quad-5.63990100$

$\begin{array}{llll}\text { C } & -2.21660100 & -2.23461500 & -3.67054900\end{array}$

$\begin{array}{llll}\text { C } & -2.08930800 & -3.62990700 & -3.43505200\end{array}$

$\begin{array}{lrrr}\mathrm{H} & -2.38130900 & -4.31712100 & -4.22375100 \\ \mathrm{C} & -1.60234400 & -4.10016900 & -2.24563100 \\ \mathrm{H} & -1.48650700 & -5.16539800 & -2.07558300 \\ \mathrm{C} & -1.28694600 & -3.19498800 & -1.21058200 \\ \mathrm{H} & -0.97841100 & -3.54589500 & -0.22745100 \\ \mathrm{C} & -1.42242400 & -1.84212300 & -1.39572300 \\ \mathrm{C} & -1.81633000 & -1.30652500 & -2.66245700 \\ \mathrm{C} & -4.58308300 & 4.48201100 & -0.77119100 \\ \mathrm{H} & -3.77791400 & 5.16899200 & -1.04561200 \\ \mathrm{C} & -5.81537300 & 4.90276300 & -1.56887900 \\ \mathrm{H} & -5.62482500 & 4.83302400 & -2.64337200 \\ \mathrm{H} & -6.05868400 & 5.94164600 & -1.32841500 \\ \mathrm{H} & -6.69258000 & 4.29453600 & -1.32695400 \\ \mathrm{C} & -4.80207900 & 4.55189300 & 0.73979300 \\ \mathrm{H} & -3.89217100 & 4.26762900 & 1.27943000 \\ \mathrm{H} & -5.60906700 & 3.88228900 & 1.05565000 \\ \mathrm{H} & -5.07480100 & 5.56991200 & 1.03333800 \\ \mathrm{C} & -5.03340500 & 1.98741300 & -1.12787100 \\ \mathrm{H} & -5.92495000 & 2.36630800 & -0.62240300 \\ \mathrm{C} & -5.43245000 & 1.52212600 & -2.52539300 \\ \mathrm{H} & -5.86067500 & 2.34368100 & -3.10603800 \\ \mathrm{H} & -6.17834600 & 0.72458700 & -2.45118400 \\ \mathrm{H} & -4.56769900 & 1.12559500 & -3.06521000\end{array}$




\begin{tabular}{|c|c|c|c|}
\hline $\mathrm{C}$ & -4.46698400 & 0.85532800 & -0.27536000 \\
\hline $\mathrm{H}$ & -4.14371500 & 1.22431400 & 0.70382600 \\
\hline $\mathrm{H}$ & -3.61667700 & 0.36975100 & -0.76513300 \\
\hline $\mathrm{H}$ & -5.23387000 & 0.09161600 & -0.11771900 \\
\hline $\mathrm{C}$ & 0.77400100 & 4.22860500 & -1.31819900 \\
\hline $\mathrm{H}$ & 1.22787900 & 5.20594200 & -1.13866600 \\
\hline $\mathrm{C}$ & 1.53039600 & 3.18791100 & -0.49554000 \\
\hline $\mathrm{H}$ & 1.59982400 & 3.49031800 & 0.55307100 \\
\hline $\mathrm{H}$ & 2.54568700 & 3.06536700 & -0.88612100 \\
\hline $\mathrm{H}$ & 1.04219400 & 2.20904800 & -0.53306000 \\
\hline $\mathrm{C}$ & 0.84309400 & 3.96438100 & -2.82156900 \\
\hline $\mathrm{H}$ & 0.27549800 & 4.71919900 & -3.37390700 \\
\hline $\mathrm{H}$ & 0.45241600 & 2.97598700 & -3.08346800 \\
\hline $\mathrm{H}$ & 1.88515900 & 4.00424200 & -3.15129800 \\
\hline $\mathrm{C}$ & -1.08331100 & 5.58054000 & -0.16846100 \\
\hline $\mathrm{H}$ & -2.07759400 & 5.34188400 & 0.21744700 \\
\hline $\mathrm{C}$ & -0.20601100 & 5.91295400 & 1.03658100 \\
\hline $\mathrm{H}$ & -0.16201500 & 5.07165600 & 1.73372000 \\
\hline $\mathrm{H}$ & -0.63522200 & 6.77067700 & 1.56194100 \\
\hline $\mathrm{H}$ & 0.81429700 & 6.18246100 & 0.74617600 \\
\hline $\mathrm{C}$ & -1.19907300 & 6.74259400 & -1.15364600 \\
\hline $\mathrm{H}$ & -1.86767600 & 6.48424200 & -1.98140900 \\
\hline $\mathrm{H}$ & -0.22164200 & 7.00509700 & -1.57225100 \\
\hline $\mathrm{H}$ & -1.59800800 & 7.62717000 & -0.64863100 \\
\hline $\mathrm{C}$ & -4.31920200 & -3.26450800 & 0.28697400 \\
\hline $\mathrm{H}$ & -3.68503200 & -2.83201000 & -0.48872600 \\
\hline $\mathrm{C}$ & -5.75423700 & -3.22643700 & -0.23324700 \\
\hline $\mathrm{H}$ & -6.08269900 & -2.19631300 & -0.40124400 \\
\hline $\mathrm{H}$ & -6.46015700 & -3.71145500 & 0.44853800 \\
\hline $\mathrm{H}$ & -5.80090800 & -3.76197800 & -1.18632500 \\
\hline $\mathrm{C}$ & -3.82692400 & -4.68231800 & 0.57465500 \\
\hline $\mathrm{H}$ & -2.82603100 & -4.65134400 & 1.01890000 \\
\hline $\mathrm{H}$ & -3.78890000 & -5.25919400 & -0.35516900 \\
\hline $\mathrm{H}$ & -4.50073200 & -5.20152900 & 1.26586700 \\
\hline $\mathrm{C}$ & -4.92435300 & -2.60970300 & 2.68658000 \\
\hline $\mathrm{H}$ & -5.73940500 & -3.27255400 & 2.38770900 \\
\hline $\mathrm{C}$ & -5.56900900 & -1.29948300 & 3.14355800 \\
\hline $\mathrm{H}$ & -6.25144300 & -0.93126900 & 2.37135200 \\
\hline $\mathrm{H}$ & -4.83123600 & -0.51464600 & 3.33436200 \\
\hline $\mathrm{H}$ & -6.13715800 & -1.45777000 & 4.06471200 \\
\hline $\mathrm{C}$ & -4.10898700 & -3.34270400 & 3.75931300 \\
\hline $\mathrm{H}$ & -3.10662000 & -2.92178500 & 3.87650500 \\
\hline $\mathrm{H}$ & -3.96817900 & -4.39019900 & 3.47989100 \\
\hline $\mathrm{H}$ & -4.62545600 & -3.30926500 & 4.72347000 \\
\hline $\mathrm{C}$ & -2.31067700 & -0.23458000 & 4.51673100 \\
\hline $\mathrm{H}$ & -3.11609900 & -0.95817500 & 4.39695000 \\
\hline $\mathrm{C}$ & -1.21236200 & -0.90330300 & 5.34338600 \\
\hline $\mathrm{H}$ & -0.82455500 & -1.77580800 & 4.80685200 \\
\hline $\mathrm{H}$ & -1.61947700 & -1.22479000 & 6.30734800 \\
\hline $\mathrm{H}$ & -0.38457300 & -0.21327100 & 5.54293100 \\
\hline $\mathrm{C}$ & -2.89845800 & 1.00809700 & 5.18115400 \\
\hline $\mathrm{H}$ & -3.68333200 & 1.44793700 & 4.55760300 \\
\hline $\mathrm{H}$ & -2.13744100 & 1.77267700 & 5.36964600 \\
\hline $\mathrm{H}$ & -3.33521900 & 0.73428200 & 6.14623800 \\
\hline $\mathrm{C}$ & -0.71228700 & 0.98171300 & 2.94821200 \\
\hline $\mathrm{H}$ & -0.56895100 & 1.48086600 & 3.90959900 \\
\hline $\mathrm{C}$ & -1.07906600 & 2.04557000 & 1.91563000 \\
\hline $\mathrm{H}$ & -1.99946000 & 2.56831400 & 2.19490100 \\
\hline $\mathrm{H}$ & -1.22583800 & 1.59423600 & 0.92792400 \\
\hline $\mathrm{H}$ & -0.27044200 & 2.77603700 & 1.82918700 \\
\hline $\mathrm{C}$ & 0.58650900 & 0.26534700 & 2.59085600 \\
\hline
\end{tabular}

$\begin{array}{lll}0.83655700 & -0.50658500 & 3.32102500\end{array}$

$\begin{array}{lll}1.40078900 & 0.99424600 & 2.54492600\end{array}$

$\begin{array}{lll}0.51245400 & -0.22674900 & 1.61838300\end{array}$

$\begin{array}{lll}1.22684200 & -0.12566600 & -1.24319800\end{array}$

$\begin{array}{lll}2.43429500 & -0.34760400 & -1.20768200\end{array}$

$\begin{array}{lll}3.15442800 & -0.47395200 & 0.12550400\end{array}$

$\begin{array}{llll}2.63121000 & 0.20457000 & 0.80693600\end{array}$

$\begin{array}{lll}4.55710600 & -0.16106500 & -0.00842900\end{array}$

$\begin{array}{lll}5.13379000 & 0.58715800 & 0.85118200\end{array}$

$\begin{array}{lll}4.44873400 & 1.20521500 & 2.03741900\end{array}$

$\begin{array}{lll}4.26887600 & 0.48256000 & 3.21881700\end{array}$

$\begin{array}{lll}3.98982300 & 2.52244400 & 1.96236900\end{array}$

$\begin{array}{lll}3.63425800 & 1.07084500 & 4.31010100\end{array}$

$\begin{array}{lll}4.63387500 & -0.53805500 & 3.28873700\end{array}$

$\begin{array}{lll}3.34035900 & 3.10392000 & 3.04788900\end{array}$

$\begin{array}{lll}4.14401000 & 3.09153800 & 1.05041300\end{array}$

$\begin{array}{lll}3.16442200 & 2.37940200 & 4.22525500\end{array}$

$\begin{array}{lll}3.50139800 & 0.50275700 & 5.22535700\end{array}$

$\begin{array}{lll}2.97972500 & 4.12590300 & 2.97547900\end{array}$

$\begin{array}{llll}2.66573500 & 2.83432300 & 5.07551900\end{array}$

$\begin{array}{lll}6.58281800 & 0.88266900 & 0.66176100\end{array}$

$\begin{array}{llll}7.32349200 & 1.53721700 & 1.65063400\end{array}$

$\begin{array}{llll}7.22278200 & 0.49625900 & -0.52345900\end{array}$

$\begin{array}{lll}8.67929700 & 1.79425000 & 1.46202800\end{array}$

$\begin{array}{lll}6.84454300 & 1.84240200 & 2.57549600\end{array}$

$\begin{array}{llll}8.57296700 & 0.75636200 & -0.71131900\end{array}$

$\begin{array}{lll}6.64177100 & -0.00566200 & -1.29016900\end{array}$

$\begin{array}{lll}9.30665200 & 1.40574600 & 0.28267700\end{array}$

$\begin{array}{llll}9.24382200 & 2.29831400 & 2.24049200\end{array}$

$\begin{array}{llll}9.05737100 & 0.45424800 & -1.63496700\end{array}$

$\begin{array}{llll}10.36341900 & 1.60688700 & 0.13501700\end{array}$

$\begin{array}{lll}3.19502800 & -0.53667000 & -2.25096400\end{array}$

$\begin{array}{lll}2.67587100 & -0.73435700 & -3.61758900\end{array}$

$\begin{array}{llll}4.36956300 & -3.12893800 & -1.07529200\end{array}$

$\begin{array}{lll}4.54245400 & -4.15736200 & -1.99626500\end{array}$

$\begin{array}{lll}3.54637600 & -5.11724800 & -2.17544100\end{array}$

$\begin{array}{lll}2.38355200 & -5.04773800 & -1.41374300\end{array}$

$\begin{array}{llll}2.21133000 & -4.01725000 & -0.49109600\end{array}$

$\begin{array}{lll}3.19407400 & -3.03923800 & -0.32054800\end{array}$

$\begin{array}{lll}5.13813000 & -2.37222200 & -0.95331600\end{array}$

$\begin{array}{lll}5.45937000 & -4.20985300 & -2.57701500\end{array}$

$\begin{array}{lll}3.68124200 & -5.91599400 & -2.89917300\end{array}$

$\begin{array}{lll}1.60549200 & -5.79650200 & -1.53378000\end{array}$

$\begin{array}{lll}1.32085400 & -3.98392100 & 0.13263800\end{array}$

$\begin{array}{llll}2.95695500 & -1.91959100 & 0.67245100\end{array}$

$\begin{array}{lll}1.94285300 & -2.01219800 & 1.07767200\end{array}$

$\begin{array}{lll}3.63874400 & -2.01170100 & 1.52556400\end{array}$

$\begin{array}{lll}-0.30870900 & -3.48590500 & 2.39574500\end{array}$

$\begin{array}{lll}1.73422400 & -1.93401500 & -3.63663100\end{array}$

$\begin{array}{lll}0.83789400 & -1.75534700 & -3.04125200\end{array}$

$\begin{array}{lll}1.42763200 & -2.12379400 & -4.67014300\end{array}$

$\begin{array}{lll}2.24168700 & -2.82383000 & -3.25337300\end{array}$

$\begin{array}{llll}2.02005100 & 0.54724900 & -4.12149400\end{array}$

$\begin{array}{lll}1.87217200 & 0.46998800 & -5.20284700\end{array}$

$\begin{array}{llll}1.04522300 & 0.71359400 & -3.66183900\end{array}$

$\begin{array}{llll}2.66160600 & 1.41135200 & -3.92271800\end{array}$

$\begin{array}{lll}3.94996800 & -1.03577000 & -4.39876000\end{array}$

$\begin{array}{lll}4.64221100 & -0.18947900 & -4.35333600\end{array}$

$\begin{array}{lll}4.44337900 & -1.91887900 & -3.98329600\end{array}$

$\begin{array}{lll}3.70420400 & -1.22980100 & -5.44679900\end{array}$ 
